\title{
Continuous matrix product states for non-relativistic quantum fields: a lattice algorithm for inhomogeneous systems
}

\author{
Martin Ganah* and Guifre Vidal \\ Perimeter Institute for Theoretical Physics, 31 Caroline Street North, Waterloo, ON N2L 2Y5, Canada
}

\begin{abstract}
By combining the continuous matrix product state (cMPS) representation for quantum fields in the continuum with standard optimization techniques for matrix product states (MPS) on the lattice, we obtain an approximation $|\Psi\rangle$, directly in the continuum, of the ground state of nonrelativistic quantum field theories. This construction works both for translation invariant systems and in the more challenging context of inhomogeneous systems, as we demonstrate for an interacting bosonic field in a periodic potential. Given the continuum Hamiltonian $H$, we consider a sequence of discretized Hamiltonians $\left\{H\left(\epsilon_{\alpha}\right)\right\}_{\alpha=1,2, \cdots, p}$ on increasingly finer lattices with lattice spacing $\epsilon_{1}>$ $\epsilon_{2}>\cdots>\epsilon_{p}$. We first use energy minimization to optimize an MPS approximation $\left|\Psi\left(\epsilon_{1}\right)\right\rangle$ for the ground state of $H\left(\epsilon_{1}\right)$. Given the MPS $\left|\Psi\left(\epsilon_{\alpha}\right)\right\rangle$ optimized for the ground state of $H\left(\epsilon_{\alpha}\right)$, we use it to initialize the energy minimization for Hamiltonian $H\left(\epsilon_{\alpha+1}\right)$, resulting in the optimized MPS $\left|\Psi\left(\epsilon_{\alpha+1}\right)\right\rangle$. By iteration we produce an optimized MPS $\left|\Psi\left(\epsilon_{p}\right)\right\rangle$ for the ground state of $H\left(\epsilon_{p}\right)$, from which we finally extract the cMPS approximation $|\Psi\rangle$ for the ground state of $H$. Two key ingredients of our proposal are: (i) a procedure to discretize $H$ into a lattice model where each site contains a two-dimensional vector space (spanned by vacuum $|0\rangle$ and one boson $|1\rangle$ states), and (ii) a procedure to map MPS representations from a coarser lattice to a finer lattice.
\end{abstract}

\section{INTRODUCTION}

Tensor networks for quantum lattice systems have their origins in the proposal twenty-five years ago of matrix product states (MPS) ${ }^{1-3}$ (introduced as finitely correlated states ${ }^{1}$ ) and the advent of the density matrix renormalization group (DMRG) ${ }^{4}$. The tensor network formalism was later on generalized using concepts and tools from quantum information theory, leading to the proposal e.g. of the multi-scale entanglement renormalization ansatz (MERA) $\sqrt{5}$ and projected entangled pair states (PEPS $)^{6}$, and is nowadays the basis of powerful variational approaches to approximate the ground-state of local lattice Hamiltonians both in one and two spatial dimensions 213. This formalism can also be used to study quantum field theories (QFTs), after a suitable lattice discretization 7 14. More recently, important proposals were made to extend tensor networks from the lattice to the continuum, so that they can be applied to quantum fields directly -that is, without resorting to a lattice discretization. Prominent examples are the continuous MPS (cMPS) ${ }^{15 / 16}$ and the continuous MERA (cMERA) $17+23$.

Ever since the original proposal of cMPS for nonrelativistic QFTs in one spatial dimension by Verstraete and Cirac ${ }^{15}$, several optimization algorithms for ground-states of translation invariant systems have been put forward $16 \mid 24 \sqrt{33}$ and applied to a number of systems. These include non-relativistic QFTs of interacting bosons $\left.{ }^{24|25| 27}|29| 32 \mid 33\right]$ and fermions ${ }^{26 \mid 30}$, and even a relativistic QFT of fermions (after modifying the kinetic term at high energies) 16 . However, in inhomogeneous (that is, non-translation invariant) systems, the cMPS formalism has proven notoriously challenging and, besides important first steps 28 , optimization algorithms have remained largely unaddressed. Only very recently, one of the authors has proposed a new, spline-based parametrization of inhomogeneous cMPS 33 and has shown how a ground- state optimization for such states can be implemented.

\section{A. Hybrid lattice/continuum strategy}

In the present manuscript we propose a hybrid lattice/continuum strategy which combines lattice MPS optimization techniques with the cMPS representation. We use this strategy to produce a cMPS approximation $|\Psi\rangle$ to the ground-state of the continuum Hamiltonian $H$ of an inhomogeneous QFT. Our central insight is that given a sufficiently fine lattice with spacing $\epsilon$, and a discretized version $H(\epsilon)$ of a continuum Hamiltonian $H$, an MPS approximation $|\Psi(\epsilon)\rangle$ to the ground state of $H(\epsilon)$ already has a hidden cMPS structure. We make use of this hidden cMPS in two different ways: (1) We exploit the cMPS representation for continuum systems to relate two MPS representations on two lattices with different lattice spacing $\epsilon$ and $\epsilon^{\prime}$. Indeed, using rescaling properties of the cMPS representation, we can translate an optimized MPS $|\Psi(\epsilon)\rangle$ for $H(\epsilon)$ into an MPS $\left|\Psi^{\prime}\left(\epsilon^{\prime}\right)\right\rangle$ on a lattice with lattice spacing $\epsilon^{\prime} \neq \epsilon$. The state $\left|\Psi^{\prime}\left(\epsilon^{\prime}\right)\right\rangle$ is of interest because it is already remarkably close to the ground-state of the Hamiltonian $H\left(\epsilon^{\prime}\right)$ on this second lattice, and thus provides an excellent starting point for an MPS energy minimization algorithm on that lattice. (2) Given the optimized MPS $|\Psi(\epsilon)\rangle$ for the ground-state of $H(\epsilon)$ for sufficiently small $\epsilon$, we can extract its hidden cMPS structure and use it to directly build a cMPS approximation $|\Psi\rangle$ for the continuum Hamiltonian $H$. The resulting cMPS approximation $|\Psi\rangle$ for the ground-state of the QFT Hamiltonian $H$ is seen to already be quite accurate. However, it is by no means an optimal cMPS approximation. Indeed, $|\Psi\rangle$ can be used as initialization of the recently introduced, spline-based, cMPS approach ${ }^{33}$, which would then produce a better cMPS approximation.

A natural question arises: if we already have an inho- 
mogeneous cMPS algorithm directly in the continuum 33 , why should we then use the proposed hybrid MPS/cMPS algorithm at all? The answer is that cMPS algorithms are generally much more delicate than lattice MPS algorithms and work best starting from a sufficiently preconverged cMPS. Indeed, as it is well documented 32 , even in the much better behaved case of translation invariant systems (both with euclidean time evolution 34 and energy minimization ${ }^{32}$ algorithms), a properly preconverged cMPS is key to preventing fatal optimization instabilities and to very significantly improve convergence time.

We have mentioned above that the cMPS representation plays an important role not just in the continuum, but also in lattices approximating the continuum, since it provides a procedure to map wave-functions between lattices with different spacing $\epsilon$ and $\epsilon^{\prime}$. As a matter of fact, the cMPS representation has inspired a new scheme for discretizing a bosonic continuum Hamiltonian $H$. A standard discretization of $H$ would lead to a boson Hubbard model where each site contains an infinite-dimensional complex vector space (corresponding to a bosonic degree of freedom). This vector space would be then truncated down to a $d$-dimensional complex vector space $\mathbb{C}_{d}$, with its $d$ levels representing the vacuum (or no-boson) state $|0\rangle$ and $n$-boson states $|n\rangle$ for $n=1,2, \cdots, d-1$. The acceptable value of $d$ on a given site might be hard to predict a priori and depends on the lattice spacing: at fixed particle density, the number of particles per site is proportional to $\epsilon$ and thus a coarser lattice requires a larger $d$ than a finer lattice. The discretization scheme we propose in this manuscript produces instead a lattice where each site is described by a two-level complex vector space $\mathbb{C}_{2}$, spanned by the vacuum state $|0\rangle$ and the one-boson state $|1\rangle$. In this case $H(\epsilon)$ can be thought of as a hard-core boson Hamiltonian with modified kinetic term. This has clear advantages over the standard discretization scheme. On the one hand, the same vector space dimension $d=2$ independent of the lattice spacing $\epsilon$ avoids having to introduce a different MPS format (with tensors of different sizes) in changing from one lattice to another. On the other hand, the cost of MPS manipulations grows with the dimension of the local vector space, and therefore using the smallest possible dimension 2 leads to lower computational times.

\section{B. Previous work on multigrid DMRG}

Our algorithm belongs to the context of multigrid MPS approaches, as pioneered by M. Dolfi, B. Bauer, M. Troyer, and Z. Ristivojevic 35 . In that work, a bosonic field on a periodic potential was also studied by first discretizing it onto a sequence of lattice Hamiltonians, and then sequentially finding an MPS approximation to the ground-state of each Hamiltonian, starting with the coarsest lattice and finishing with the finest one, and using the optimized MPS on one lattice to build an ini- tial MPS for the optimization on the next, finer lattice. As the authors there demonstrated $\sqrt{35}$, a multi-grid MPS approach successfully circumvents the problem one faces when trying to directly optimize an MPS on the finest lattice, namely that the optimization gets stuck in local minima with an incorrect spatial profile of particle density. Our approach thus shares important similarities with this proposal. However, it also contains several significant improvements, based on exploiting the cMPS representation hidden in a lattice MPS, which we outline next.

On the one hand, while both approaches have as input a continuum Hamiltonian $H$, the multi-grid approach of Ref ${ }^{[5}$ outputs an approximate ground-state wave-function on some fine lattice, whereas our hybrid algorithm outputs an approximate ground-state wavefunction directly back in the continuum. This is not just a more natural format for the output (recall that the lattice was introduced only as a computational device), but one which may lead to easier comparison with other continuum approaches, such as perturbative methods 36 , Bethe Ansatz ${ }^{37 \mid 38}$ or the Gross-Pitaevskii approach 39 [4]

A second major difference is in how the transition from a coarser lattice to a finer lattice takes place in the two approaches. Dolfi et al ${ }^{35}$ use an ad hoc MPS splitting procedure that is essentially independent of the actual wave-function under consideration. Instead, by extracting and exploiting the approximate cMPS structure hidden in a lattice MPS, we will see that our approach produces a better pre-converged initial state on the finer lattice. In addition, the hidden cMPS description allows for much more flexibility in how the coarser and finer lattice relate. While the method proposed by Dolfi et al ${ }^{35}$ is restricted to mapping one site to $n$ sites, here we can map $m$ sites to $n$ sites for any pair of integers $m, n-$ and one can even consider transitions between two irregular lattice discretizations (where both coarser and finer lattices have a lattice spacing that depends on position). Such flexibility is relevant when studying inhomogeneous systems with e.g. a position-dependent potential $V(x)$ that changes faster in some places than others, since it allows us to locally adjust the lattice spacing to the needs of the problem.

Finally, a third important difference is that Dolfi et al. ${ }^{35}$ used a standard scheme to discretize the continuum bosonic Hamiltonian $H$ into a lattice Hamiltonian on a lattice where each site had a complex vector space truncated to $d=3$ levels, whereas we propose and use a discretization scheme that produces $d=2$-level sites, and thus potentially smaller computational times.

\section{Outline}

The rest of the manuscript is organized into the following sections:

In Sect. II we review necessary background material, including standard discretization of a continuous Hamil- 
tonian $H$ into a lattice, and the cMPS and MPS variational states. In the remaining sections we present our proposed algorithm, together with a demonstration of its performance. More specifically, in Sect. III we explain how to extract a hidden, approximate cMPS representation from an MPS, and how to discretize a continuous, bosonic Hamiltonian $H$ into a two-level lattice model. In Sect. IV we describe the algorithm that takes as input a continuum Hamiltonian $H$ and produces a cMPS approximation $|\Psi\rangle$ for its ground-state, with technical details described in the Appendix. In Sect. V we present results for a concrete system, and in Sect. VI we compare our approach to the multi-grid method proposed by Dolfi et al. $[35$.

\section{BACKGROUND MATERIAL}

In this section we review the type of QFT Hamiltonian $H$ whose ground-state we would like to investigate, its discretization onto a sequence of lattice Hamiltonians $H\left(\epsilon_{\alpha}\right)$, as well as the continuous MPS to approximate ground-states directly in the continuum and the matrix product state (MPS) to approximate ground-states of the lattice.

\section{A. Hamiltonian in the continuum}

For the sake of concreteness, in this manuscript we focus on a specific inhomogeneous Hamiltonian for a nonrelativistic bosonic field of the form

$$
\begin{aligned}
H & =\frac{1}{2 m} \int d x \partial_{x} \psi^{\dagger}(x) \partial_{x} \psi(x)+\int d x \mu(x) \psi^{\dagger}(x) \psi(x) \\
& +g \int d x \psi^{\dagger}(x) \psi^{\dagger}(x) \psi(x) \psi(x)
\end{aligned}
$$

which describes the dynamics of a single species of a bosonic field on the real line. The creation operator $\psi(x)$ fulfills the canonical bosonic field commutation relations

$$
\left[\psi(x), \psi^{\dagger}(y)\right]=\delta(x-y) .
$$

Above, $\frac{1}{2 m} \int d x \partial_{x} \psi^{\dagger}(x) \partial_{x} \psi(x)$ is the non-relativistic kinetic term, with $m$ the mass of a boson, $g \int d x \psi^{\dagger}(x) \psi^{\dagger}(x) \psi(x) \psi(x)$ is a quartic local interaction term with interaction strength $g$ and $\int d x \mu(x) \psi^{\dagger}(x) \psi(x)$ is a chemical potential term. In this work we will focus on the case of a periodic chemical potential of the form

$$
\mu(x)=\mu_{0}+V_{0}\left(\cos \left(\frac{2 \pi x}{L}\right)-1\right)^{2}
$$

parametrized by offset $\mu_{0}$, amplitude $V_{0}$ and periodicity $L$. More generally, one could consider a similar Hamiltonian involving multi-species of bosons, single species or multiple species of fermionic fields, and even a mixture of bosonic and fermionic fields.

\section{B. Hamiltonian on the lattice}

Hamiltonian $H$ in Eq. (1) can be discretized following a standard procedure. First, the real line $\mathbb{R}$ is replaced by a lattice $\mathcal{L}(\epsilon)$ of equidistant points $\left\{x_{i} \equiv \epsilon i\right\}_{i \in \mathbb{Z}}$, with lattice spacing $\epsilon \equiv x_{i+1}-x_{i}$. At each position $x_{i}$, one then replaces the bosonic annihilation operator $\psi(x)$ in the continuum with a bosonic annihilation operator $\psi\left(x_{i}\right)$ defined as

$$
\psi(x) \rightarrow \psi\left(x_{i}\right) \equiv \frac{1}{\sqrt{\epsilon}} c_{i} .
$$

$c_{i}$ is a bosonic lattice annihilation operator at site $i$ fulfilling the canonical bosonic lattice commutation relations

$$
\left[c_{i}, c_{j}^{\dagger}\right]=\delta_{i j}
$$

Using the standard replacements $\int d x \rightarrow \epsilon \sum_{i}$ and $\partial_{x} f(x) \rightarrow(f(x+\epsilon)-f(x)) / \epsilon$, the non-relativistic kinematic term becomes

$$
\begin{aligned}
& \frac{1}{2 m} \int d x \partial_{x} \psi^{\dagger}(x) \partial_{x} \psi(x) \rightarrow \\
& \quad-\frac{1}{2 m \epsilon^{2}} \sum_{i}\left(c_{i}^{\dagger} c_{i+1}+\text { h.c. }\right)+\frac{1}{m \epsilon^{2}} \sum_{i} c_{i}^{\dagger} c_{i},
\end{aligned}
$$

whereas the chemical potential term transforms into

$$
\int d x \mu(x) \psi^{\dagger}(x) \psi(x) \rightarrow \sum_{i} \mu_{i} c_{i}^{\dagger} c_{i}
$$

with $\mu_{i} \equiv \mu\left(x_{i}\right)$, and the interaction term reads

$$
g \int d x \psi^{\dagger}(x) \psi^{\dagger}(x) \psi(x) \psi(x) \rightarrow \frac{g}{\epsilon} \sum_{i} c_{i}^{\dagger} c_{i}^{\dagger} c_{i} c_{i}
$$

We therefore end up with the lattice Hamiltonian

$$
\begin{aligned}
& H(\epsilon) \equiv-\frac{1}{2 m \epsilon^{2}} \sum_{i}\left(c_{i}^{\dagger} c_{i+1}+\text { h.c. }\right) \\
& \quad+\sum_{i}\left(\mu_{i}+\frac{1}{m \epsilon^{2}}\right) c_{i}^{\dagger} c_{i}+\frac{g}{\epsilon} \sum_{i} c_{i}^{\dagger} c_{i}^{\dagger} c_{i} c_{i} .
\end{aligned}
$$

We emphasize that later on we will propose a more convenient, alternative discretization scheme which results in modified kinetic and interaction terms, see Eq. (46), acting on a lattice $\mathcal{L}(\epsilon)$ where each site is described by a two-dimensional complex vector space spanned by $|0\rangle$ and $|1\rangle$, that is $d=2$, for any value of $\epsilon$.

Hamiltonian $H(\epsilon)$ acts on a lattice where each site $i$ is described by an infinite-dimensional complex vector space spanned by states $\left\{\left|n_{i}\right\rangle\right\}$, for $n_{i}=0,1,2, \cdots$, where the state $\left|n_{i}\right\rangle$ fulfills $c_{i}^{\dagger} c_{i}\left|n_{i}\right\rangle=n_{i}\left|n_{i}\right\rangle$ and thus corresponds to having $n_{i}$ bosonic particles on that site. In practical calculations one must truncate this basis to a finite number $d$ of states, so that $n_{i}=0,1, \cdots, d-1$. In a ground-state calculation, this approximation may be well justified (see below). 


\section{Scaling of expectation values with $\epsilon$}

Let us assume that the ground-state expectation value of the particle density $\langle n(x)\rangle \equiv\left\langle\psi^{\dagger}(x) \psi(x)\right\rangle$, the quartic term $\left\langle\psi^{\dagger}(x) \psi^{\dagger}(x) \psi(x) \psi(x)\right\rangle$, and and the kinetic term $\left\langle\partial_{x} \psi^{\dagger}(x) \partial_{x} \psi(x)\right\rangle$ are finite and that the corresponding lattice expectation values converge smoothly to the continuum. This implies that, for small lattice spacing $\epsilon$ and to leading order in $\epsilon$, we have

$$
\begin{aligned}
& \left\langle c_{i}^{\dagger} c_{i}\right\rangle \approx \epsilon\left\langle\psi^{\dagger}(x) \psi(x)\right\rangle=O(\epsilon), \\
& \left\langle c_{i}^{\dagger} c_{i}^{\dagger} c_{i} c_{i}\right\rangle \approx \epsilon^{2}\left\langle\psi^{\dagger}(x) \psi^{\dagger}(x) \psi(x) \psi(x)\right\rangle=O\left(\epsilon^{2}\right), \\
& \left\langle\left(c_{i+1}-c_{i}\right)^{\dagger}\left(c_{i+1}-c_{i}\right)\right\rangle \approx \\
& \qquad \epsilon^{3}\left\langle\partial_{x} \psi^{\dagger}(x) \partial_{x} \psi(x)\right\rangle=O\left(\epsilon^{3}\right) .
\end{aligned}
$$

In particular, if $P_{n_{i}} \equiv\left\langle\mid n_{i}\right\rangle\left\langle n_{i} \mid\right\rangle$ is the probability that site $i$ is found in state $\left|n_{i}\right\rangle$ when the lattice is in the ground-state of $H(\epsilon)$, with $\sum_{n_{i}} P_{n_{i}}=1$ and $P_{n_{i}} \geq 0$, then we have $\left\langle c_{i}^{\dagger} c_{i}\right\rangle=\sum_{n_{i}} n_{i} P_{n_{i}},\left\langle c_{i}^{\dagger} c_{i}^{\dagger} c_{i} c_{i}\right\rangle=$ $\sum_{n_{i}} n_{i}\left(n_{i}-1\right) P_{n_{i}}$ and Eqs. (11)-(12) imply

$$
\begin{aligned}
O(\epsilon) & =\sum_{n_{i}=0}^{\infty} n_{i} P_{n_{i}} \geq \sum_{n_{i}=1}^{\infty} P_{n_{i}} \\
O\left(\epsilon^{2}\right) & =\sum_{n_{i}=0}^{\infty} n_{i}\left(n_{i}-1\right) P_{n_{i}} \geq \sum_{n_{i}=2}^{\infty} P_{n_{i}} .
\end{aligned}
$$

Eq. (14) tells us that the probability $P_{1}$ of finding one or more boson on one site is at most $O(\epsilon)$. Eq. 115 implies that the probability $P_{2}$ of finding two or more bosons at one site is at most $O\left(\epsilon^{2}\right)$. If we were to truncate the Hilbert space dimension to $d=2$, then we would be throwing away contributions of order $\epsilon^{2}$. However, this approximation would be incompatible with the interaction term in the Hamiltonian Eq. (1), which requires at least two bosons on one site, that is $d=3$. We conclude that a valid truncation of the local Hilbert space requires at least $d=3$.

\section{Continuous matrix product states}

The continuous matrix product state (cMPS) $15 \mid 42$ is a variational ansatz for ground-states of non-relativistic QFT Hamiltonians such as $H$ in Eq.(1). On the real line it has the form

$$
|\Psi\rangle=v_{l}^{\dagger} \mathcal{P} e^{\int_{-\infty}^{\infty} d x Q(x) \otimes \mathbb{1}+R(x) \otimes \psi^{\dagger}(x)} v_{r}|0\rangle,
$$

where for each value $x \in \mathbb{R}, Q(x), R(x) \in \mathbb{C}^{D \times D}$ are $D \times D$ matrices of complex coefficients, $\mathcal{P} e$ denotes a path ordered exponential, $|0\rangle$ is the physical vacuum, that is $\psi(x)|0\rangle=0 \quad \forall x \in \mathbb{R}$, and $v_{l}, v_{r}$ are arbitrary boundary vectors at $x= \pm \infty$.

The matrix functions $Q(x)$ and $R(x)$ contain the variational parameters of the ansatz. The expectation value of local observables can be expressed in terms of these matrices. For instance, we have

$$
\begin{aligned}
& \left\langle\psi^{\dagger}(x) \psi(x)\right\rangle=\langle l(x)|R(x) \otimes \bar{R}(x)| r(x)\rangle \\
& \left\langle\psi^{\dagger}(x) \psi^{\dagger}(x) \psi(x) \psi(x)\right\rangle=\left\langle l(x)\left|R^{2}(x) \otimes \bar{R}^{2}(x)\right| r(x)\right\rangle \\
& \left\langle\partial_{x} \psi^{\dagger}(x) \partial_{x} \psi(x)\right\rangle=\langle l(x)|\left([Q(x), R(x)]+\frac{d R(x)}{d x}\right) \\
& \otimes\left([\bar{Q}(x), \bar{R}(x)]+\frac{d \bar{R}(x)}{d x}\right)|r(x)\rangle
\end{aligned}
$$

where $\langle l(x)|$ and $|r(x)\rangle$ are $D^{2}$-dimensional vectors that depend on the cMPS description at smaller and larger values of $x$, respectively, and fulfill $\langle l(x) \mid r(x)\rangle=\langle\Psi \mid \Psi\rangle=$ 1. In particular, $\langle l(x)|$ and $|r(x)\rangle$ can be chosen to have only finite entries and therefore finite matrices $Q(x)$ and $R(x)$ and $d R(x) / d x$ will guarantee a finite value for Eqs. 17) -19 .

\section{E. Lattice matrix product states}

The MPS is a variational ansatz for ground-states of lattice Hamiltonians such as $H(\epsilon)$ in Eq. 10p. It has the form

$$
|\Psi\rangle=\sum_{\cdots, n_{i}, n_{i+1}, \cdots} \cdots A_{n_{i}}^{[i]} A_{n_{i+1}}^{[i+1]} \cdots\left|\cdots n_{i} n_{i+1} \cdots\right\rangle
$$

where $i$ labels lattice sites, and $n_{i}=0,1 \cdots, d-1$ labels states $\left|n_{i}\right\rangle$ of the occupation number basis on that site. $A_{n_{i}}^{[i]} \in \mathbb{C}^{D \times D}$ is a $D \times D$ matrix of complex coefficients. We often refer to the set of $d$ matrices $\left\{A_{0}^{[i]}, A_{1}^{[i]}, \cdots, A_{d-1}^{[i]}\right\}$ on site $i$ as a three-index MPS tensor and denote it as $A^{[i]}$.

The MPS tensors $A^{[i]}$ for all sites $i \in \mathbb{Z}$ contain the variational parameters of this ansatz.

\section{F. Periodic potential}

In this work we consider that the chemical potential $\mu(x)$ in the continuum Hamiltonian $H$ in Eq. (1) is a periodic function,

$$
\mu(x+L)=\mu(x) .
$$

We will then assume that the ground-state of $H$ has the same periodicity, which we will incorporate into the cMPS through the periodicity conditions

$$
Q(x+L)=Q(x), \quad R(x+L)=R(x) .
$$

By choosing a lattice spacing $\epsilon$ such that $L / \epsilon \equiv N \in \mathbb{N}$, the discretized Hamiltonian $H(\epsilon)$ in Eq. 10 is periodic under shifts by $N$ sites, with the chemical potential $\mu_{i}$ fulfilling $\mu_{i+N}=\mu_{i}$. In that case, if the ground state is also periodic under shifts of $N$ sites, we can restrict 
out attention to an MPS with a unit cell consisting of $N$ MPS tensors $\left\{A^{[i]}, A^{[i+1]}, \cdots, A^{[i+N-1]}\right\}$, with

$$
A^{[i+N]}=A^{[i]} .
$$

Several results presented in the next sections do not rely on having a periodic potential.

\section{G. cMPS as the continuous limit of an MPS}

The cMPS wave function Eq. 16. can be understood 15 as the continuum limit of a continuous family of MPS $A^{[i]}(\epsilon)$ parametrized by the lattice spacing $\epsilon$ of the lattice $\mathcal{L}(\epsilon)$ and defined as

$$
\begin{array}{ll}
A_{0}^{[i]}(\epsilon) \equiv \mathbb{1}+\epsilon Q\left(x_{i}\right) & n_{i}=0, \\
A_{n_{i}}^{[i]}(\epsilon) \equiv \sqrt{\frac{\epsilon^{n_{i}}}{n_{i} !}}\left[R\left(x_{i}\right)\right]^{n_{i}} & n_{i} \geq 1,
\end{array}
$$

where $Q\left(x_{i}\right)$ and $R\left(x_{i}\right)$ are the cMPS matrices $Q(x)$ and $R(x)$ evaluated at $x=x_{i}$. Indeed, in the limit $\epsilon \rightarrow 0$ one can recover the cMPS decomposition in Eq. 16) from the MPS decomposition in Eq. 2015.

The particular form of the MPS tensors $A^{[i]}$ in Eq. 24. Eq. 25) ensures that expectation values of local operators such as $\left\langle c_{i}^{\dagger} c_{i}\right\rangle,\left\langle c_{i}^{\dagger} c_{i}^{\dagger} c_{i} c_{i}\right\rangle$, and $\left\langle\left(c_{i+1}-c_{i}\right)^{\dagger}\left(c_{i+1}-c_{i}\right)\right\rangle$ are continuous as a function of the lattice spacing $\epsilon$ and scale as in Eqs. 111)- 13 for small values of $\epsilon$.

\section{H. Gauge freedom}

The cMPS representation contains a gauge freedom, meaning that there are redundant parameters. If $Q(x), R(x)$ is a cMPS representing a state $|\Psi\rangle$, then the pair $\tilde{Q}(x)$ and $\tilde{R}(x)$ given by

$$
\begin{aligned}
\tilde{Q}(x) & =g(x) Q(x) g(x)^{-1}-\frac{d g(x)}{d x} g(x)^{-1} \\
\tilde{R}(x) & =g(x) R(x) g(x)^{-1}
\end{aligned}
$$

is another cMPS that represents the same state $|\Psi\rangle$. Here $g(x)$ is some invertible $D \times D$ matrix. We can use this freedom to impose certain conditions on the cMPS matrices. In this work we will mostly work on the left canonical gauge, characterized by

$$
Q^{\dagger}(x)+Q(x)+R^{\dagger}(x) R(x)=0,
$$

for which the $D^{2}$-dimensional left vector $\langle l(x)|$ and right vector $|r(x)\rangle$ become the identity operator and a diagonal density matrix, after they are reorganized as $D \times D$ matrices.

Analogously, on the lattice the MPS representation $A^{[i]}$ and $\tilde{A}^{[i]}$ represent the same state $|\Psi\rangle$ if they are related by

$$
\tilde{A}^{[i]}=g^{[i-1]} A^{[i]}\left(g^{[i]}\right)^{-1},
$$

where, for all $i \in \mathbb{Z}$, the $D \times D$ matrix $g^{[i]}$ is invertible. The left canonical gauge reads

$$
\sum_{n_{i}}\left(A_{n_{i}}^{[i]}\right)^{\dagger} A_{n_{i}}^{[i]}=\mathbb{1}, \quad \sum_{n_{i}} A_{n_{i}}^{[i]} \rho\left(A_{n_{i}}^{[i]}\right)^{\dagger}=\rho
$$

where $\rho$ is a diagonal density matrix containing the squares of the Schmidt values $\lambda_{\alpha}$, i.e.

$$
[\rho]_{\alpha \beta}=\lambda_{\alpha}^{2} \delta_{\alpha \beta}
$$

\section{EXPLOITING THE CMPS REPRESENTATION ON THE LATTICE}

In this section we explain how to extract a cMPS representation from an MPS that approximates the groundstate on a fine lattice. We then propose a modified discretization scheme that produces discrete Hamiltonians $H(\epsilon)$ on a lattice made of two-level sites. Finally, we explain how to use the hidden cMPS representation to map an MPS on one lattice to an MPS on another lattice.

\section{A. Extraction of a cMPS from a lattice MPS}

Given a lattice MPS $|\Psi(\epsilon)\rangle$ that approximates the ground-state of $H(\epsilon)$, we extract cMPS matrices $Q(x)$ and $R(x)$ evaluated on the points $x_{i} \in \mathcal{L}(\epsilon)$ through

$$
\begin{aligned}
Q\left(x_{i}\right) & \equiv \frac{A_{0}^{[i]}-\mathbb{1}}{\epsilon}, \\
R\left(x_{i}\right) & \equiv \frac{A_{1}^{[i]}}{\sqrt{\epsilon}},
\end{aligned}
$$

and then extend these matrices $Q\left(x_{i}\right)$ and $R\left(x_{i}\right)$ from $\mathcal{L}(\epsilon)$ to the real line by interpolation (see below).

It is important to recognize that $Q\left(x_{i}\right)$ depends nontrivially on the choice of gauge in the MPS. Indeed, if $\tilde{A}^{[i]}$ relates to $A^{[i]}$ through Eq. $(29)$, then it is easy to check that for $\tilde{Q}\left(x_{i}\right) \equiv\left(\tilde{A}_{0}^{[i]}-\mathbb{1}\right) / \epsilon$ we get

$$
\begin{aligned}
\tilde{Q}\left(x_{i}\right) & =\frac{1}{\epsilon}\left(g^{[i-1]} A^{[i]}\left(g^{[i]}\right)^{-1}-\mathbb{1}\right) \\
& \neq \frac{1}{\epsilon} g^{[i-1]}\left(A^{[i]}-\mathbb{1}\right)\left(g^{[i]}\right)^{-1} \\
& =g^{[i-1]} Q\left(x_{i}\right)\left(g^{[i]}\right)^{-1}
\end{aligned}
$$

which means that the final cMPS state $|\Psi\rangle$ extracted from the MPS state $|\Psi(\epsilon)\rangle$ is affected by the specific gauge choice we work with. In particular, a random choice of gauge may result in matrices $Q\left(x_{i}\right)$ and $R\left(x_{i}\right)$ that change abruptly with $x_{i}$. This typically leads to a cMPS that is not as useful for our current purposes. We have heuristically determined that it is best to work with a diagonal gauge, such as e.g. the left canonical gauge of Eq. (30), which we use from now on (see Appendix A 
for a more detailed explanation). In particular, interpolation of the matrices $Q\left(x_{i}\right), R\left(x_{i}\right)$ is carried out in this diagonal gauge. To extend the extracted state to the continuum, we use a standard basis-spline interpolation on the matrix entries $\left[Q\left(x_{i}\right)\right]_{\alpha \beta},\left[R\left(x_{i}\right)\right]_{\alpha \beta}$. For brevity, let $M \in\{Q, R\}$ label the two different matrices in the following. Each interpolation takes as input a set of data points $\left(x_{i},\left[M\left(x_{i}\right)\right]_{\alpha \beta}\right)$. A continuous and smooth function $f_{\alpha \beta}^{M}(x)$ is then constructed such that $f_{\alpha \beta}^{M}\left(x_{i}\right) \equiv\left[M\left(x_{i}\right)\right]_{\alpha \beta}$. The function $f_{\alpha \beta}^{M}(x)$ is chosen as an expansion in so-called order $k$ basis-spline polynomials $B_{i}^{k}(x)$, i.e.

$$
f_{\alpha \beta}^{M}(x)=\sum_{i} B_{i}^{k}(x) \mathcal{F}_{\alpha \beta}^{M, i}
$$

with properly chosen expansion coefficients $\mathcal{F}_{\alpha \beta}^{M, \sqrt{33 \mid 43}}$ The polynomials $B_{i}^{k}(x)$ have finite support on a small region inside the interval $[0, L]$, with $L$ the periodicity of the Hamiltonian $H$. We use order $k=5$ polynomials in this manuscript, and use periodic spline interpolation. The cMPS matrix functions are then given by

$$
\begin{aligned}
& Q_{\alpha \beta}(x)=f_{\alpha \beta}^{Q}(x) \\
& R_{\alpha \beta}(x)=f_{\alpha \beta}^{R}(x)
\end{aligned}
$$

The cMPS matrices $Q(x)$ and $R(x)$ extracted from an MPS are useful for a number of purposes. For instance, when working on a lattice $\mathcal{L}(\epsilon)$ with sufficiently small lattice spacing $\epsilon$, the extracted cMPS may already provide a good approximation to the ground-state of the continuum Hamiltonian $H$.

\section{B. Mapping an MPS from $\mathcal{L}(\epsilon)$ to $\mathcal{L}\left(\epsilon^{\prime}\right)$}

On the other hand, we can use the extracted cMPS matrices $Q(x)$ and $R(x)$ and Eqs. (24)-(25) to define a new state $\left|\Psi^{\prime}\left(\epsilon^{\prime}\right)\right\rangle$ on another lattice $\mathcal{L}\left(\epsilon^{\prime}\right)$ with lattice spacing $\epsilon^{\prime} \neq \epsilon$, such that site $i$ in $\mathcal{L}(\epsilon)$ and in $\mathcal{L}\left(\epsilon^{\prime}\right)$ is on positions $x_{i}$ and $x_{i}^{\prime}$, respectively, with

$$
x_{i} \equiv \epsilon i, \quad x_{i}^{\prime} \equiv \epsilon^{\prime} i .
$$

Indeed, we can define MPS tensors for $\left|\Psi^{\prime}\left(\epsilon^{\prime}\right)\right\rangle$ according to

$$
\begin{array}{ll}
A_{0}^{\prime[i]} \equiv \mathbb{1}+\epsilon^{\prime} Q\left(x_{i}^{\prime}\right) & n_{i}=0, \\
A_{n_{i}}^{\prime[i]} \equiv \sqrt{\frac{\epsilon^{\prime n_{i}}}{n_{i} !}}\left[R\left(x_{i}^{\prime}\right)\right]^{n_{i}} & n_{i} \geq 1
\end{array}
$$

so that, overall, we map $|\Psi(\epsilon)\rangle$ into $\left|\Psi^{\prime}\left(\epsilon^{\prime}\right)\right\rangle$ through three steps

$$
\begin{array}{ccl}
A^{[i]} & \stackrel{\text { Eqs. }}{\longrightarrow}, \sqrt[31]{\longrightarrow} & Q\left(x_{i}\right), R\left(x_{i}\right) \\
& \stackrel{\text { interpolation }}{\longrightarrow} & Q(x), R(x) \\
& \stackrel{\text { Eqs. 38, } 39]}{\longrightarrow} & A^{\prime[i] .}
\end{array}
$$

\section{Modified discretization}

Recall that on the lattice we can truncate the infinitedimensional vector space on each site down to $d$ dimensions, where $d$ depends on the lattice spacing $\epsilon$. Recall also that the cost of an MPS computation grows with $d$, and therefore it would be convenient to work with the smallest non-trivial local space, which corresponds to $d=2$. It would therefore be of interest if we could discretize the continuum Hamiltonian $H$ into a lattice Hamiltonian $H(\epsilon)$ acting on a lattice where each site has a vector space of dimension $d=2$. It turns out that by thinking in terms of the cMPS decomposition, this is possible.

To make a local Hilbert space dimension of $d=2$ compatible with a bosonic theory, the discretized kinetic and interaction energy operators have to be modified. Let us first discuss the necessary modifications for the kinetic energy operator. For illustration purposes, let us for a moment consider a translation invariant MPS $|\Psi\rangle$ with tensors $A_{n_{i}}^{[i]}(\epsilon)$ of the form

$$
\begin{aligned}
A_{0}^{[i]}(\epsilon) & =\mathbb{1}+\epsilon Q & & n_{i}=0 \\
A_{n_{i}}^{[i]}(\epsilon) & =\sqrt{\frac{\epsilon^{n_{i}}}{n_{i} !}}[R]^{n_{i}} & & n_{i} \geq 1 .
\end{aligned}
$$

However, the following results are equally valid for an inhomogeneous state.

Let us recall that the action of the annihilation operator $c_{i}$ on the state $|\Psi\rangle$ is given by

$$
c_{i}|\Psi\rangle=\cdots A_{n_{i-1}}^{[i-1]}\left[\sqrt{\epsilon} R|0\rangle+\mathcal{O}\left(\epsilon^{3 / 2}\right)\right] A_{n_{i+1}}^{[i+1]} \ldots .
$$

If we compute the action of the discretized derivative operator $\frac{1}{\epsilon^{3 / 2}}\left(\mathbb{1}_{i} c_{i+1}-c_{i} \mathbb{1}_{i+1}\right)$ on this state, we obtain, to lowest orders in $\epsilon$, an expression of the form 


$$
\begin{aligned}
& \frac{1}{\epsilon^{3 / 2}}\left(\mathbb{1}_{i} c_{i+1}-c_{i} \mathbb{1}_{i+1}\right)|\Psi\rangle= \\
& \cdots A_{n_{i-1}}^{[i-1]} \frac{1}{\epsilon^{3 / 2}}\left[(\mathbb{1}+\epsilon Q) \sqrt{\epsilon} R|00\rangle+(\mathbb{1}+\epsilon Q) \sqrt{\frac{\epsilon^{2}}{2}} \sqrt{2} R^{2}|01\rangle+\epsilon R^{2}|10\rangle\right. \\
& \left.-\sqrt{\epsilon} R(\mathbb{1}+\epsilon Q)|00\rangle-\sqrt{\frac{\epsilon^{2}}{2}} \sqrt{2} R^{2}(\mathbb{1}+\epsilon Q)|10\rangle-\epsilon R^{2}|01\rangle+\mathcal{O}\left(\epsilon^{2}\right)\right] A_{n_{i+2}}^{[i+2]} \ldots \\
& =\cdots A_{n_{i-1}}^{[i-1]} \frac{1}{\epsilon^{3 / 2}}[\underbrace{((\mathbb{1}+\epsilon Q) \sqrt{\epsilon} R-\sqrt{\epsilon} R(\mathbb{1}+\epsilon Q))}_{\epsilon^{3 / 2}[Q, R]}|00\rangle+ \\
& \underbrace{\left(\epsilon R^{2}-\sqrt{\frac{\epsilon^{2}}{2}} \sqrt{2} R^{2}\right)}_{0}|10\rangle+\underbrace{\left(\epsilon R^{2}-\sqrt{\frac{\epsilon^{2}}{2}} \sqrt{2} R^{2}\right)}_{0}|01\rangle+\mathcal{O}\left(\epsilon^{2}\right)] A_{n_{i+2}}^{[i+2]} \ldots .
\end{aligned}
$$

Here $\left|n_{i} n_{i+1}\right\rangle \equiv\left|n_{i}\right\rangle \otimes\left|n_{i+1}\right\rangle$ are occupation number states. All terms of order $\epsilon$ inside the square brackets are seen to cancel, leaving a leading term $\propto \epsilon^{3 / 2}$ which cancels with the pre-factor $\frac{1}{\epsilon^{3 / 2}}$ to produce a finite result. Crucially, the cancellations of order $\epsilon$ involve basis states $\left|n_{i}=2\right\rangle$ and $\left|n_{i+1}=2\right\rangle$. A similar calculation for the case of $d=2$ will result in a non-vanishing contribution of order $\epsilon$, which will diverge in the limit $\epsilon \rightarrow 0$ (unless $R^{2}=0$ ). Thus, a Hilbert space dimension of $d=2$ seems irreconcilable with a bosonic theory. However, if we modify the term $\frac{1}{\epsilon^{3 / 2}}\left(\mathbb{1}_{i} c_{i+1}-c_{i} \mathbb{1}_{i+1}\right)$ to

$$
\frac{1}{\epsilon^{3 / 2}}\left(\mathbb{1}_{i} c_{i+1}-c_{i} \mathbb{1}_{i+1}\right) \rightarrow \frac{1}{\epsilon^{3 / 2}}\left(\mathcal{P}_{i}^{0} c_{i+1}-c_{i} \mathcal{P}_{i+1}^{0}\right),
$$

with $\mathcal{P}_{i}^{0}$ a projection operator onto the empty state $|0\rangle$ at position $x_{i}$, it is easy to verify that all terms of order $\epsilon$ will vanish. Furthermore, the result of the operator application $\frac{1}{\epsilon^{3 / 2}}\left(\mathcal{P}_{i}^{0} c_{i+1}-c_{i} \mathcal{P}_{i+1}^{0}\right)|\Psi\rangle$ is, to lowest order in $\epsilon$, seen to be identical to Eq. (43). Similarly, the operator $c_{i} c_{i}$ in the interaction is not compatible with a local Hilbert space dimension of $d=2$. To overcome this problem, we make the replacement

$$
c_{i} c_{i} \rightarrow c_{i} c_{i+1}
$$

Similar to the kinetic energy operator, the result of $c_{i} c_{i+1}|\Psi\rangle$ is, to lowest order in $\epsilon$, seen to be identical to that of $c_{i} c_{i}|\Psi\rangle$. After these replacements the final lattice Hamiltonian $H$ assumes the form

$$
\begin{aligned}
& H(\epsilon) \equiv-\frac{1}{2 m \epsilon^{2}} \sum_{i}\left(c_{i}^{\dagger} c_{i+1}+\text { h.c. }\right) \\
& +\frac{1}{2 m \epsilon^{2}} \sum_{i}\left(\mathcal{P}_{i}^{0} c_{i+1}^{\dagger} c_{i+1}+c_{i}^{\dagger} c_{i} \mathcal{P}_{i+1}^{0}\right) \\
& +\sum_{i} \mu_{i} c_{i}^{\dagger} c_{i} \\
& +\frac{g}{\epsilon} \sum_{i} c_{i}^{\dagger} c_{i+1}^{\dagger} c_{i} c_{i+1} .
\end{aligned}
$$

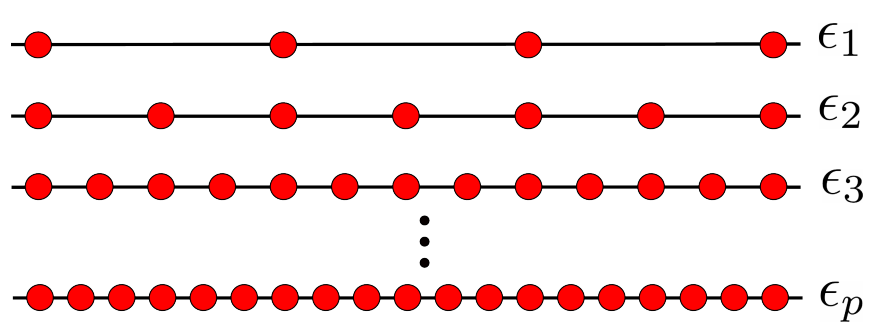

FIG. 1: Consecutive fine-graining of a lattice. For each lattice spacing $\epsilon_{\alpha}$ we obtain a Hamiltonian $H\left(\epsilon_{\alpha}\right)$ from Eq. 46.

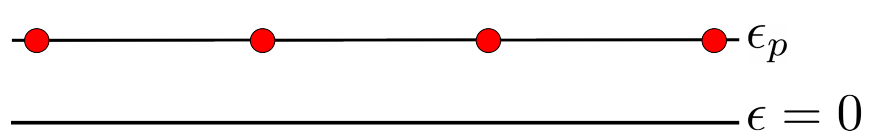

FIG. 2: Obtaining a cMPS from a lattice MPS. We extract the cMPS matrices $\left\{Q\left(x_{i}\right), R\left(x_{i}\right)\right\}$ from the groundstate $\left|\Psi\left(\epsilon_{p}\right)\right\rangle$ of a lattice optimization and use interpolation techniques to obtain continuous matrices $Q(x), R(x)$ (see Sec III A.

and the MPS tensors are given by

$$
\begin{aligned}
& A_{0}^{[i]}(\epsilon)=\mathbb{1}+\epsilon Q \\
& A_{1}^{[i]}(\epsilon)=\sqrt{\epsilon} R .
\end{aligned}
$$

\section{A HYBRID MPS/CMPS ALGORITHM}

In this section we describe an algorithm that takes a continuous, inhomogeneous Hamiltonian $H$ of a nonrelativistic QFT, and produces a cMPS approximation for its ground-state. 

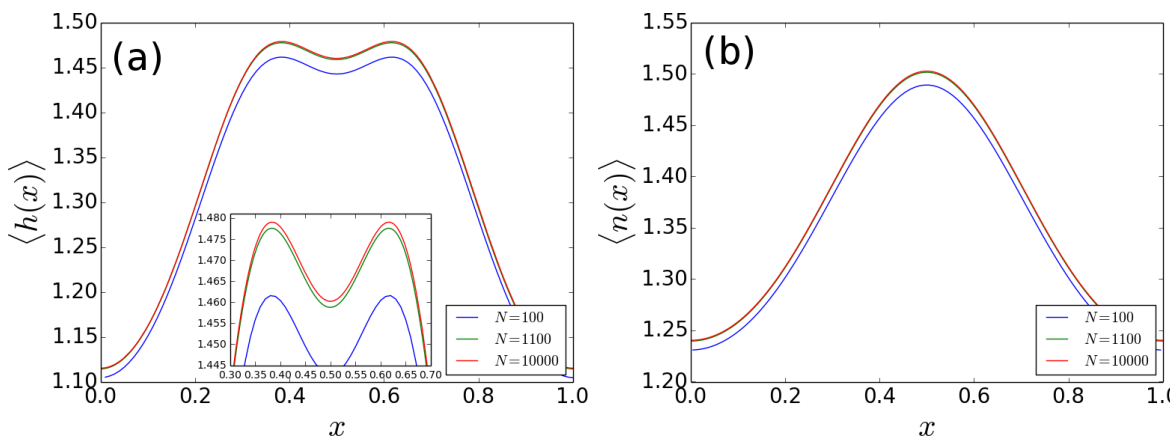

FIG. 3: Ground-state observables for different lattices. $\left\langle\frac{1}{2 x} \partial_{x} \psi^{\dagger}(x) \partial_{x} \psi(x)+g \psi^{\dagger}(x) \psi^{\dagger}(x) \psi(x) \psi(x)\right\rangle$ of the ground-state of $\mathrm{Eq}$-1 for $D$ for different number of lattice points per unit-cell. (b) Particle density $\langle n(x)\rangle$ and (c) inte, $\mu_{0}=-0.5, V_{0}=-1.0, g=1.0$, same ground-states as in (a).

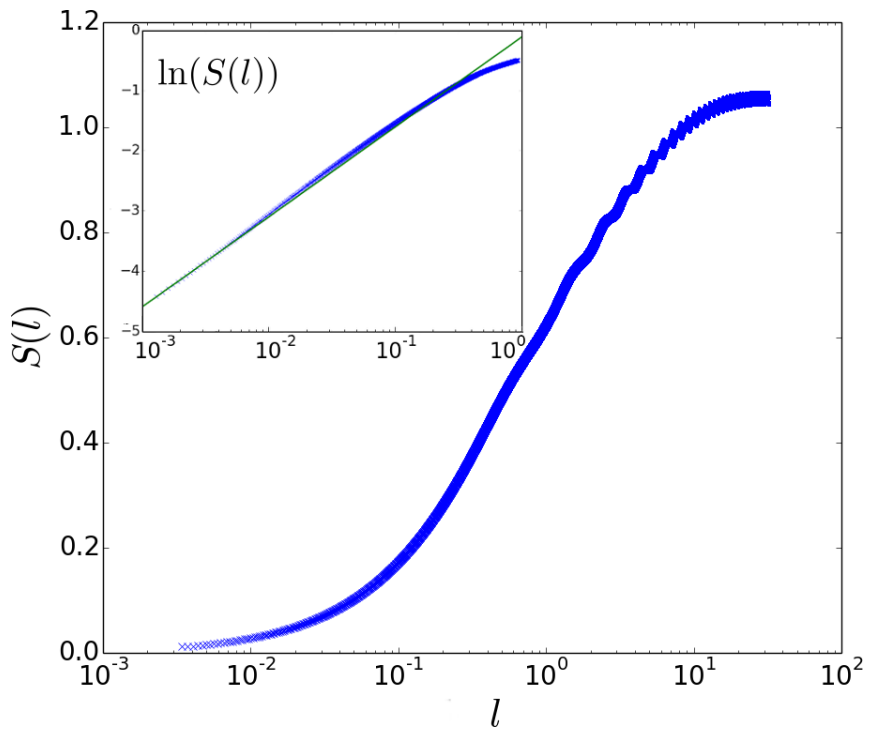

FIG. 4: Entanglement entropy of a bulk region of length $\boldsymbol{l}$. Main figure: entanglement entropy $S(l)$ in the ground-state of Eq. (46) of a small region of length $l$ as a function of $l$, for $D=16, \mu_{0}=-0.5, V_{0}=-1.0, g=1.0, L=1.0$, and $N=10^{4}$ lattice points per unit-cell. Oscillations are due to the periodicity of the state. Inset: $\ln (S(l))$ vs. $\log (l)$. For the smallest $l$ visible, we see a power law increase in $S(l)$ which levels off for larger $l>10^{-1}$. The point where the curvature in the figure changes sign is approximately at $l \approx 0.05$. The green line is a fit to the first 15 data-points.

\section{A. Sequence of lattice Hamiltonians}

To obtain a cMPS approximation $|\Psi\rangle$ for the groundstate of the continuum Hamiltonian $H$, we first construct a sequence of lattice Hamiltonians $H\left(\epsilon_{\alpha}\right), \alpha=1 \ldots p$, with $\epsilon_{1}>\epsilon_{2} \cdots>\epsilon_{p}$. This is schematically represented in Fig. 1. We then sequentially compute an MPS approximation $\left|\Psi\left(\epsilon_{\alpha}\right)\right\rangle$ to the ground-state of Hamiltonian $H\left(\epsilon_{\alpha}\right)$. We initialize the ground-state optimization of $H\left(\epsilon_{\alpha}\right)$ using the extension of $\left|\Psi\left(\epsilon_{\alpha-1}\right)\right\rangle$ to the lattice

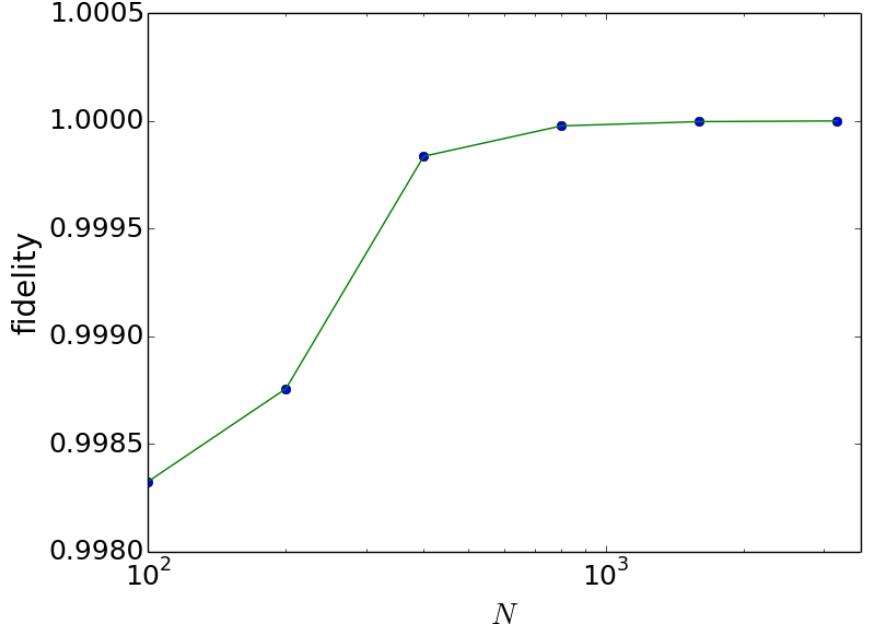

FIG. 5: Quality of interpolation. Fidelity per unit-cell of ground-states on $N=100,200,400,800,1600$ and 3200 grid points per unit-cell after interpolation to $N_{\text {final }}=3200$ with ground-state at $N_{\text {final }}=3200$. Parameters are the same as in Fig. 3 Lines are guides to the eye.

$\mathcal{L}\left(\epsilon_{\alpha}\right)$, as described above. $\left|\Psi\left(\epsilon_{1}\right)\right\rangle$ is initialized with random, constant matrices $Q, R$. From the converged state $\left|\Psi\left(\epsilon_{p}\right)\right\rangle$ we then construct a cMPS approximation $|\Psi\rangle$ to the ground-state of the continuum Hamiltonian $H$, see Fig. 2.

\section{B. Optimization of MPS with a finite unit-cell}

For each Hamiltonian $H\left(\epsilon_{\alpha}\right)$ we obtain the groundstate from am infinite DMRG (iDMRG) method, as introduced by McCulloch $\frac{4144}{4}$, extended to the case of a large unit-cell with $N \gg 2$ sites. In Appendix D we present in detail the implementation for the cases considered in this manuscript, and explain possible variants of the algorithm, including a gradient-based optimization method for translation invariant lattices. Once the ground-state $\left|\Psi\left(\epsilon_{\alpha}\right)\right\rangle$ of $H\left(\epsilon_{\alpha}\right)$ has been found, we extract 

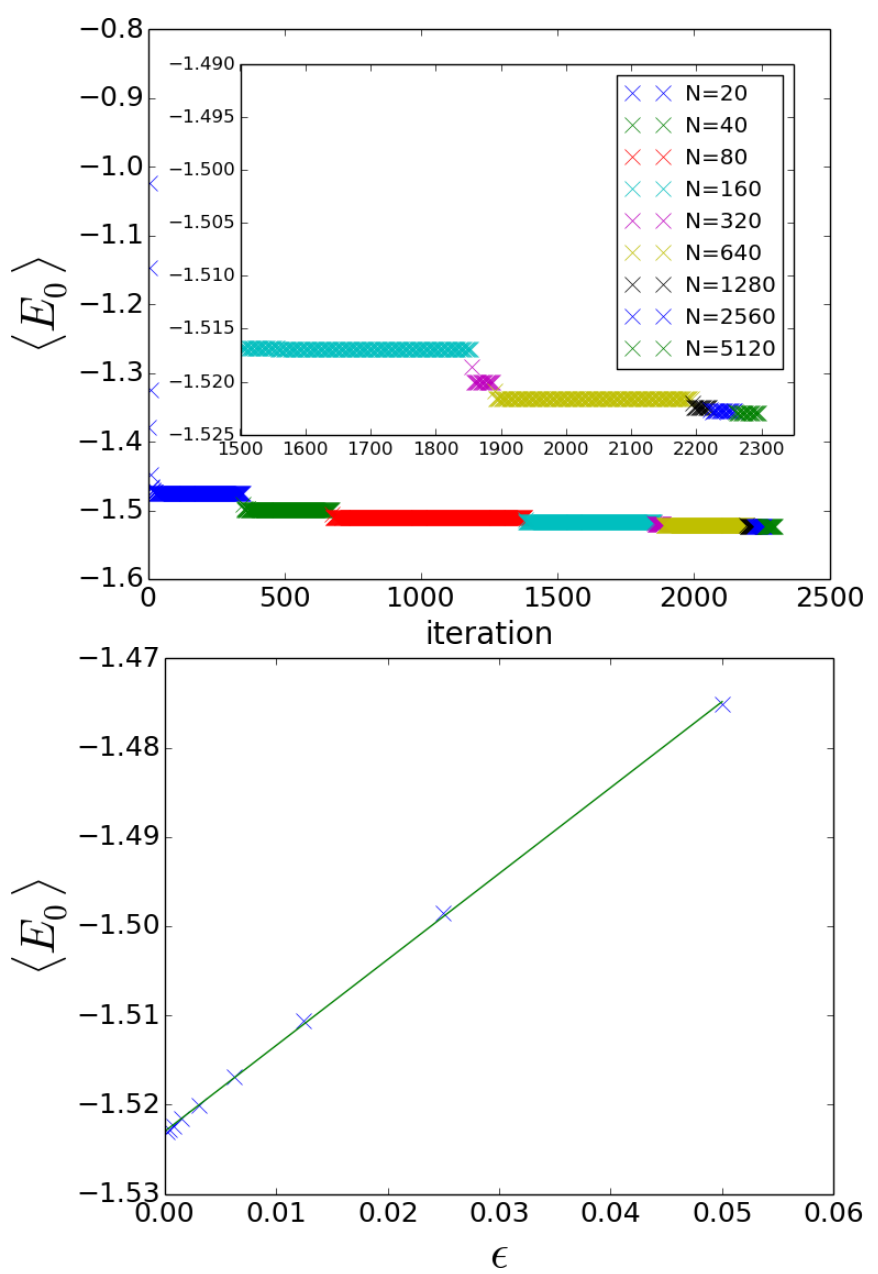

FIG. 6: Convergence of the ground-state energy. Upper panel: ground-state energy per unit-cell, as a function of DMRG sweeps for $\mu_{0}=-0.5, V_{0}=-1.0, g=1.0, D=$ $32, L=1.0$. The different colours correspond to different finegraining. At each lattice spacing, ground-state energies are converged to $10^{-8}$. Blue crosses at small iteration numbers are for $N=20$ lattice sites; following crosses are obtained by taking the MPS from the previous discretization and using a spline interpolation on the matrices $Q\left(x_{i}\right), R\left(x_{i}\right)$ to obtain a finer discretization, and continue optimization for the finegrained lattice. Lower panel: converged ground-state energy per unit-cell for different grid spacings $\epsilon$.

the cMPS matrices $\left\{Q\left(x_{i}\right), R\left(x_{i}\right)\right\}$ and interpolate them to obtain an initial state $\left|\Psi^{\prime}\left(\epsilon_{\alpha+1}\right)\right\rangle$ for the optimization of the next Hamiltonian $H\left(\epsilon_{\alpha+1}\right)$ (see Secs. IIIA and IIIB. Finally, after having obtained the groundstate $\left|\Psi\left(\epsilon_{p}\right)\right\rangle$ of $H\left(\epsilon_{p}\right)$, we use an interpolation to extract a cMPS approximation $Q(x), R(x)$ to the continuous ground-state, see Sec. III A

\section{EXAMPLE}

In this section we present results for ground-states observables of Eq.46) with a periodic background potential
Eq.(3) with periodicity $L=1$. In particular, we show results for lattices $\mathcal{L}\left(\epsilon=\frac{L}{N}\right)$ with an increasing number $N$ of discretization points per unit-cell. For all calculations, we set $m=0.5, g=1.0, \mu_{0}=-0.5$ and $V_{0}=-1.0$. On each grid $\mathcal{L}\left(\frac{L}{N}\right)$ we run the optimization until the energy per unit-cell is converged to $10^{-8}$. In Fig. 3 (a), (b) and (c) we compare converged energy densities $\langle h(x)\rangle \equiv\left\langle\frac{1}{2 m} \partial_{x} \psi^{\dagger}(x) \partial_{x} \psi(x)+g \psi^{\dagger}(x) \psi^{\dagger}(x) \psi(x) \psi(x)\right\rangle$, particle densities $\langle n(x)\rangle$ and interaction term $\left\langle\left[\psi^{\dagger}(x)\right]^{2} \psi^{2}(x)\right\rangle$ from optimization on three different grids $N=100$ (blue line), $N=1100$ (green line) and $N=10000$ (red line), obtained by successive interpolation from $N=100$ to $N=1100$, and from $N=1100$ to $N=10000$. We observe a clear convergence with increasing number of discretization points (the inset in Fig. 3 (a) zooms into the central region of the figure).

For large lattice spacing $\epsilon \geq 0.05$, we find that the interpolation method tends to perform worse than for small $\epsilon$. Qualitatively, this follows from a dependence of the lattice matrices $Q\left(x_{i}, \epsilon\right), R\left(x_{i}, \epsilon\right)$ on the discretization $\epsilon$. For large $\epsilon \geq 0.05$, we find that an interpolation to e.g. $\epsilon^{\prime}=\epsilon / 2$ and successive normalization of the MPS introduces changes in $Q\left(x_{i}, \epsilon\right), R\left(x_{i}, \epsilon\right)$ which are comparable to $Q\left(x_{i}, \epsilon\right), R\left(x_{i}, \epsilon\right)$. Thus, interpolation changes drastically the state. However, once the discretization is fine enough such that $\epsilon$ is well below a transition scale $\xi$ (see below), corrections to $Q\left(x_{i}, \epsilon\right), R\left(x_{i}, \epsilon\right)$ from interpolation become much smaller than $Q\left(x_{i}, \epsilon\right), R\left(x_{i}, \epsilon\right)$, and interpolation in this case gives accurate ansatz states for finer grids. This can quantitatively be seen from analyzing for example the behaviour of the entanglement entropy $S(l)=-\operatorname{tr} \rho(l) \log (\rho(l))$ of a bulk region of length $l$, where $\rho(l)$ is the reduced density matrix of this region. In Fig. 4 we show $S(l)$ for a ground-state on a very fine grid with $N=10^{4}$ discretization points. Oscillations are due to the periodicity of the state. The inset shows $S(l)$ on a log-log scale. $S(l)$ exhibits a transition from a power law increase for $l<0.05$ to a weaker increase for $l>0.05$. This transition length scale $\xi \approx 0.05$ coincides with the region of the breakdown of the interpolation method, and depends on the location of the region $l$ within the unit-cell (see Appendix $\mathrm{C}$ ). The presence of a space dependent effective cutoff $\xi=\xi(x)$ suggests that it can be used to create optimal discretization grids for MPS by adjusting the grid distances $\epsilon(x)$ to $\xi(x)$. Note that this effective cutoff can be similarly seen in other physical observables like e.g. the superconducting correlation function $\left\langle\psi^{\dagger}(l) \psi(0)\right\rangle$ (not shown).

An appealing feature of the presented method is the fact that it gives access to a parametrization of the ground-state of an interacting quantum field theory in terms of a set of smooth functions $Q(x)$ and $R(x)$. This opens up new possible applications of lattice MPS methods. As described above, one application is the extension by interpolation of the ground state at one discretization to a new state at an arbitrarily finer discretization. Even though in general this new state will not be the ground-state of the finer lattice, it will be a good ap- 
proximation to it, which can be used to initialize an optimization on the finer lattice. In particular, interpolation becomes an increasingly soft perturbation as one fine-grains the lattice. This is illustrated in Fig. 5, where we plot the fidelity per unit-cell of states obtained by interpolating the ground-states $\left|\Psi\left(\epsilon_{\alpha}\right)\right\rangle$ of lattices $\mathcal{L}\left(\epsilon_{\alpha}\right)$ with $N_{\alpha}=\{100,200,400,800,1600\}, \epsilon_{\alpha}=\frac{L}{N_{\alpha}}$ to a very fine lattice $\mathcal{L}\left(\epsilon_{\text {final }}\right)$ with $N_{\text {final }}=3200, \epsilon_{\text {final }}=\frac{L}{N_{\text {final }}}$. Each ground-states $\left|\Psi\left(\epsilon_{\alpha}\right)\right\rangle$ is obtained using a standard iDMRG optimization, and then extended to a state $\left|\Psi_{\alpha}^{\prime}\left(\epsilon_{\text {final }}\right)\right\rangle$ on $\mathcal{L}\left(\epsilon_{\text {final }}\right)$. We then calculate the fidelity per unit-cell of the overlap $\left\langle\Psi_{\alpha}^{\prime}\left(\epsilon_{\text {final }}\right) \mid \Psi\left(\epsilon_{\text {final }}\right)\right\rangle$ As can be seen from the figure, the fidelity rapidly converges towards 1.0, indicating that the interpolated, nonoptimized state $\left|\Psi_{\alpha}^{\prime}\left(\epsilon_{\text {final }}\right)\right\rangle$ is very close to the true ground-state of the final lattice (see also Appendix B for further discussion on interpolation).

As we argue above, the speed of convergence of an optimization on a given lattice $\mathcal{L}\left(\epsilon^{\prime}\right)$ can be drastically improved by a proper choice of initialization. To this end, we use the proposed interpolation method to extend the MPS $|\Psi(\epsilon)\rangle$ from a previous optimization to the current lattice $\mathcal{L}\left(\epsilon^{\prime}\right)$. The resulting state $\left|\Psi^{\prime}\left(\epsilon^{\prime}\right)\right\rangle$ is then used as initial state to the optimization on $\mathcal{L}\left(\epsilon^{\prime}\right)$. The merit of this approach is demonstrated in the upper panel of Fig. 6. where we show the ground-state energy per unitcell as a function of the iteration number in the optimization (each iteration corresponds to a DMRG sweep over the unit-cell). After the energy is converged within $10^{-8}$ we take the state, prolong it to a finer grid and continue optimization. Different colors in the figure correspond to different number of lattice sites $N$ per unit-cell. The inset zooms onto the right part of the figure. The lower panel in Fig. 6 shows the ground-state energy per unitcell as a function of discretization parameter $\epsilon$, with a clear linear behaviour in $\epsilon$.

\section{DISCUSSION}

As we have already mentioned in the introduction, our proposed method shares important similarities with the multigrid DMRG method proposed by Dolfi et al. 35 . In this section we give a quantitative comparison between the multigrid approach and our proposed interpolation method. We start with a quick summary of the multigrid approach.

To transform an MPS from a lattice with $N$ sites to a lattice with $N^{\prime}=K N$ sites, with $K \in \mathbb{N}$, the multigrid approach splits up each tensor $A_{n_{i}}^{[i]}$ into a product of $K$ new tensors $\tilde{A}_{\tilde{n}_{1}}^{\left[i_{1}^{\prime}\right]} \ldots \tilde{A}_{\tilde{n}_{K}}^{\left[i_{K}^{\prime}\right]}$. The splitting is performed by first contracting $A_{n_{i}}^{[i]}$ with a tensors $T_{\tilde{n}_{1}, \ldots, \tilde{n}_{K}}^{n_{i}}$,

$$
T_{\tilde{n}_{1}, \ldots, \tilde{n}_{K}}^{n_{i}}=\frac{\delta\left(n_{i}, \sum_{\alpha=1}^{K} \tilde{n}_{\alpha}\right)}{\sqrt{\sum_{n_{1}^{\prime}, \ldots} \delta\left(n_{i}, \sum_{\alpha=1}^{K} n_{\alpha}^{\prime}\right)}},
$$

and then using an SVD to decompose the resulting tensor

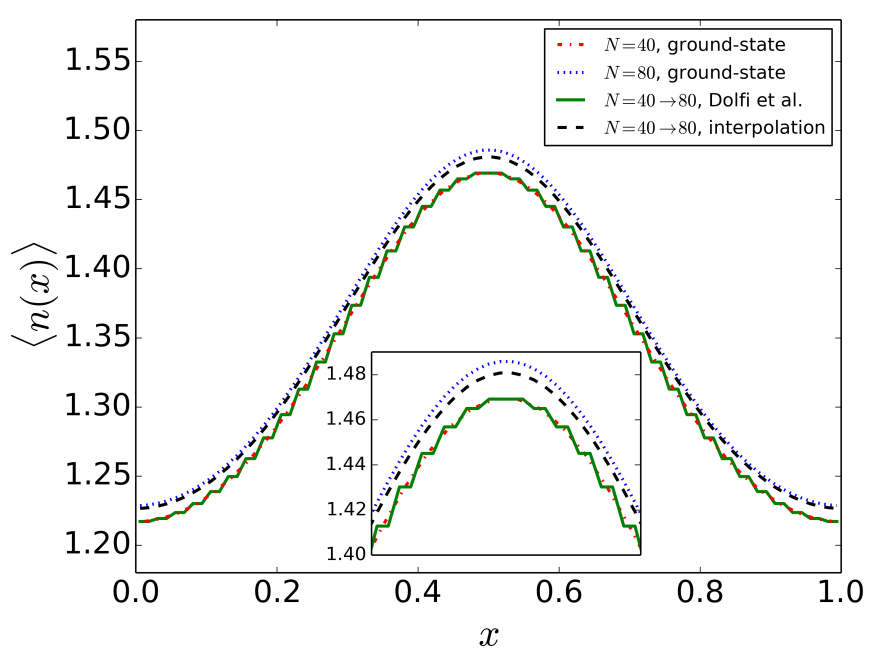

FIG. 7: Particle density $\langle\boldsymbol{n}(\boldsymbol{x})\rangle$ for different interpolation schemes. Starting from the ground-state of lattice with $N=40$ per unit-cell (red dash-dotted line), we use prolongation as proposed by Dolfi et al. (green solid line) and our proposed interpolation method (black dashed line) to obtain a state on a finer lattice with $N=80$ sites per unitcell. For comparison we also show $\langle n(x)\rangle$ for the optimized ground-state on $N=80$ sites per unit-cell. The inset shows a magnification of the central peak.

into a product of $K$ tenors $\tilde{A}_{\tilde{n}_{1}}^{\left[i_{1}^{\prime}\right]} \ldots \tilde{A}_{\tilde{n}_{K}}^{\left[i_{K}^{\prime}\right]}$. For the case of $K=2$, this is graphically represented as

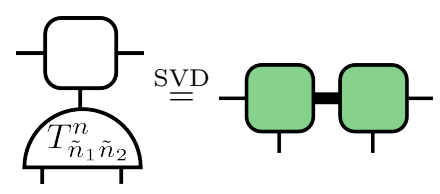

(see the Appendix for a short summary of diagrammatic MPS notations). Note that the bond dimension between the two new tensors has increased, which we have indicated by drawing a thicker line between the two.

To compare the multigrid approach with our interpolation method, we start from a ground-state on a lattice with $N=40$ sites per unit-cell, and extend this state to a grid with $N=80$ sites per unit-cell, using the multigrid approach and our proposed interpolation method. In Fig. 7 we show results for the particle density $\left\langle n\left(x_{i}\right)\right\rangle$ for the different approaches. The red dash-dotted line shows $\left\langle n\left(x_{i}\right)\right\rangle$ for the ground-state on $N=40$ lattice sites per unit-cell. The green solid and black dashed line are the results from the extension to a lattice with $N=80$ sites using a multigrid approach and our interpolation method, respectively. The blue dotted line is the groundstate for the $N=80$ site lattice. From this result we see that the multigrid approach already gives a good ansatz state. However, the prolongation here produces step-like artifacts. The interpolation on the other hand produces a smooth density profile which is seen to be already very close to the true ground-state density profile on a lattice with $N=80$ sites per unit-cell. The inset shows a zoom onto the central region. Note that the total particle 
number is not fixed in our model.

\section{CONCLUSION}

We have presented a method which unifies the continuous matrix product state representation for quantum fields with standard optimization techniques for MPS on the lattice. Our method is equally applicable to both translation invariant and inhomogeneous systems, as we demonstrate for an interacting bosonic field in a periodic potential. Starting from a continuum Hamiltonian $H$, we construct a sequence of discretized Hamiltonians $\left\{H\left(\epsilon_{\alpha}\right)\right\}_{\alpha=1,2, \cdots, p}$ on increasingly finer lattices $\mathcal{L}\left(\epsilon_{\alpha}\right)$ with lattice spacings $\epsilon_{1}>\epsilon_{2}>\cdots>\epsilon_{p}$. Our method is initialized by using energy minimization for lattice MPS to optimize an MPS approximation $\left|\Psi\left(\epsilon_{1}\right)\right\rangle$ to the ground-state of $H\left(\epsilon_{1}\right)$. We then use the MPS $\left|\Psi\left(\epsilon_{\alpha}\right)\right\rangle$ optimized for the ground state of $H\left(\epsilon_{\alpha}\right)$ to initialize the energy minimization for Hamiltonian $H\left(\epsilon_{\alpha+1}\right)$, resulting in the optimized MPS $\left|\Psi\left(\epsilon_{\alpha+1}\right)\right\rangle$. To initialize the optimization we make use of the hidden cMPS structure of the MPS $\left|\Psi\left(\epsilon_{\alpha}\right)\right\rangle$ to extend it from the lattice $\mathcal{L}\left(\epsilon_{\alpha}\right)$ to the lattice $\mathcal{L}\left(\epsilon_{\alpha+1}\right)$. From the final MPS $\left|\Psi\left(\epsilon_{p}\right)\right\rangle$ for the ground state of $H\left(\epsilon_{p}\right)$, we then extract the cMPS approximation $|\Psi\rangle$ for the ground state of $H$ directly in the continuum. For the variational energy optimization, we introduce a new procedure to discretize $H$ into a lattice model where each site contains a two-dimensional vector space (spanned by vacuum $|0\rangle$ and one boson $|1\rangle$ states). Our method can be generalized to the case of multiple species of bosons or fermions, or even mixtures of both.

\section{Acknowledgements}

The authors thank J. Rincón, F. Verstraete and D. Draxler for insightful discussions. The authors also acknowledge support by the Simons Foundation (Many Electron Collaboration). Computations were made on the supercomputer Mammouth parallèle II from University of Sherbrooke, managed by Calcul Québec and Compute Canada. The operation of this supercomputer is funded by the Canada Foundation for Innovation (CFI), the ministère de l'Économie, de la science et de l'innovation du Québec (MESI) and the Fonds de recherche du Québec - Nature et technologies (FRQ-NT). This research was supported in part by Perimeter Institute for Theoretical Physics. Research at Perimeter Institute is supported by the Government of Canada through Industry Canada and by the Province of Ontario through the Ministry of Economic Development \& Innovation.
* corresponding author: martin.ganahl@gmail.com

1 M. Fannes, B. Nachtergaele, and R. F. Werner, Communications in Mathematical Physics 144, 443 (1992).

${ }^{2}$ U. Schollwöck, Annals of Physics 326, 96 (2011).

${ }^{3}$ F. Verstraete, J. I. Cirac, and V. Murg, arXiv:0907.2796 (2009).

${ }^{4}$ S. R. White, Physical Review Letters 69, 2863 (1992).

${ }^{5}$ G. Vidal, Physical Review Letters 99, 220405 (2007).

${ }^{6}$ F. Verstraete and J. I. Cirac, arXiv:cond-mat/0407066 (2004).

7 E. M. Stoudenmire, L. O. Wagner, S. R. White, and K. Burke, Physical Review Letters 109, 056402 (2012).

8 A. Milsted, J. Haegeman, and T. J. Osborne, Physical Review D 88, 085030 (2013).

9 L. O. Wagner, E. M. Stoudenmire, K. Burke, and S. R. White, Physical Review Letters 111 (2013).

${ }^{10}$ L. O. Wagner, T. E. Baker, E. M. Stoudenmire, K. Burke, and S. R. White, Physical Review B 90, 045109 (2014).

11 T. E. Baker, E. M. Stoudenmire, L. O. Wagner, K. Burke, and S. R. White, Physical Review B 91, 235141 (2015).

12 T. E. Baker, E. M. Stoudenmire, L. O. Wagner, K. Burke, and S. R. White, Physical Review B 93, 119912(E) (2016).

13 E. M. Stoudenmire and S. R. White, Physical Review Letters 119, 046401 (2017)

14 T. E. Baker, K. Burke, and S. R. White, arXiv:1709.03460 [physics] (2017).

15 F. Verstraete and J. I. Cirac, Physical Review Letters 104, 190405 (2010).

16 J. Haegeman, J. I. Cirac, T. J. Osborne, H. Verschelde, and F. Verstraete, Physical Review Letters 105, 251601 (2010).
17 J. Haegeman, T. J. Osborne, H. Verschelde, and F. Verstraete, Physical Review Letters 110, 100402 (2013).

18 M. Miyaji, T. Numasawa, N. Shiba, T. Takayanagi, and K. Watanabe, Physical Review Letters 115, 171602 (2015).

19 Q. Hu and G. Vidal, Physical Review Letters 119, 010603 (2017).

20 A. Franco-Rubio and G. Vidal, arXiv:1706.02841 [condmat, physics:hep-th, physics:quant-ph] (2017).

21 X. Wen, G. Y. Cho, P. L. S. Lopes, Y. Gu, X.-L. Qi, and S. Ryu, Physical Review B 94, 075124 (2016).

22 J. Molina-Vilaplana, Journal of High Energy Physics 2015 , $2(2015)$

23 J. S. Cotler, J. Molina-Vilaplana, and M. T. Mueller, arXiv:1612.02427 [hep-th, physics:quant-ph] (2016).

24 D. Draxler, J. Haegeman, T. J. Osborne, V. Stojevic, L. Vanderstraeten, and F. Verstraete, Physical Review Letters 111, 020402 (2013).

${ }^{25}$ F. Quijandría, J. J. García-Ripoll, and D. Zueco, Physical Review B 90, 235142 (2014).

26 S. S. Chung, K. Sun, and C. J. Bolech, arXiv:1501.00228 [cond-mat, physics:quant-ph] (2014).

27 F. Quijandría and D. Zueco, Physical Review A 92, 043629 (2015).

28 J. Haegeman, D. Draxler, V. Stojevic, J. I. Cirac, T. J. Osborne, and F. Verstraete, arXiv:1501.06575 [cond-mat, physics:hep-th, physics:math-ph, physics:nlin, physics:quant-ph] (2015).

29 J. Rincón, M. Ganahl, and G. Vidal, Physical Review B 92, 115107 (2015)

30 S. S. Chung, K. Sun, and C. J. Bolech, Physical Review B 91, 121108 (2015). 
31 D. Draxler, J. Haegeman, F. Verstraete, and M. Rizzi, arXiv:1609.09704 [cond-mat, physics:quant-ph] (2016).

32 M. Ganahl, J. Rincón, and G. Vidal, Physical Review Letters 118, 220402 (2017).

33 M. Ganahl, arXiv:1712.01260 [cond-mat, physics:physics] (2017).

34 J. Haegeman, J. I. Cirac, T. J. Osborne, I. Pižorn, H. Verschelde, and F. Verstraete, Physical Review Letters 107, 070601 (2011)

35 M. Dolfi, B. Bauer, M. Troyer, and Z. Ristivojevic, Physical Review Letters 109, 020604 (2012).

36 M. A. Cazalilla, Journal of Physics B: Atomic, Molecular and Optical Physics 37, S1 (2004).

37 E. H. Lieb and W. Liniger, Physical Review 130, 1605 (1963).

38 E. H. Lieb, Physical Review 130, 1616 (1963).

39 L. P. Pitaevskii, Journal of Experimental and Theoretical Physics 13, 451 (1961).

40 E. P. Gross, Il Nuovo Cimento (1955-1965) 20, 454 (1961).

41 E. P. Gross, Journal of Mathematical Physics 4, 195 (1963).

42 J. Haegeman, J. I. Cirac, T. J. Osborne, and F. Verstraete, Physical Review B 88, 085118 (2013).

43 H. Bachau, E. Cormier, P. Decleva, J. E. Hansen, and F. Martín, Reports on Progress in Physics 64, 1815 (2001).

44 I. P. McCulloch, 0804.2509 (2008).

45 R. Ors and G. Vidal, Physical Review B 78, 155117 (2008).

46 E. M. Stoudenmire and S. R. White, Physical Review B 87, 155137 (2013).

47 V. Zauner-Stauber, L. Vanderstraeten, M. T. Fishman, F. Verstraete, and J. Haegeman, arXiv:1701.07035 [condmat, physics:quant-ph] (2017).

\section{Appendix A: Shifting of the orthogonality center and regauging}

In the following we describe how to shift the orthogonality center of an MPS. For the discrete lattice case presented in this manuscript, we use a QR decomposition on the tensors $A^{[i]}$ to shift the orthogonality center. However, to keep the cMPS form explicit, that is keep matrices in the form Eq. 47), we use an approach which is slightly different from the standard procedure. The generic form of an MPS after a local optimization step at site $i+1$ is

$\cdots\left(\begin{array}{c}\mathbb{1}+\epsilon Q^{l}\left(x_{i}\right) \\ \sqrt{\epsilon} R^{l}\left(x_{i}\right)\end{array}\right)\left(\begin{array}{c}V\left(x_{i+1}\right) \\ \sqrt{\epsilon} R\left(x_{i+1}\right)\end{array}\right)\left(\begin{array}{c}\mathbb{1}+\epsilon Q^{r}\left(x_{i+2}\right) \\ \sqrt{\epsilon} R^{r}\left(x_{i+2}\right)\end{array}\right) \ldots$

where superscripts $l / r$ indicate left or right orthonormal matrices ${ }^{33 / 42}$. Pulling out $V\left(x_{i+1}\right)$ yields

$$
\begin{array}{r}
\cdots\left(\begin{array}{c}
\mathbb{1}+\epsilon Q^{l}\left(x_{i}\right) \\
\sqrt{\epsilon} R^{l}\left(x_{i}\right)
\end{array}\right)\left(\begin{array}{c}
\mathbb{1} \\
\sqrt{\epsilon} R\left(x_{i+1}\right)\left[V\left(x_{i+1}\right)\right]^{-1}
\end{array}\right) V\left(x_{i+1}\right) \\
\times\left(\begin{array}{c}
\mathbb{1}+\epsilon Q^{r}\left(x_{i+2}\right) \\
\sqrt{\epsilon} R^{r}\left(x_{i+2}\right)
\end{array}\right) \cdots .
\end{array}
$$

Using a QR decomposition, the center term in brackets is then orthonormalized:

$$
\left(\begin{array}{c}
\mathbb{1} \\
\sqrt{\epsilon} R\left(x_{i+1}\right)\left[V\left(x_{i+1}\right)\right]^{-1}
\end{array}\right) \stackrel{Q R}{\rightarrow}\left(\begin{array}{c}
\mathbb{1}+\epsilon Q^{l}\left(x_{i+1}\right) \\
\sqrt{\epsilon} R^{l}\left(x_{i+1}\right)
\end{array}\right) C\left(x_{i+1}\right) .
$$

The matrix product $C\left(x_{i+1}\right) V\left(x_{i+1}\right)$ is then normalized and absorbed into the matrices at site $i+2$. A similar procedure is applied to shift the center site to the left. Crucially, we find that the standard QR algorithm in numpy, equipped with a phase-convention for the diagonal of $R$ (not be confused with the cMPS matrix), preserves the cMPS form of the tensors. Note that due to the gauge freedom of (c)MPS, the matrices $R\left(x_{i}\right)$ and $Q\left(x_{i}\right)$ do not need to be continuous, even in the limit $\epsilon \rightarrow 0$. For a random initial state, we observe that during the local updates, matrices $Q\left(x_{i}\right)$ and $R\left(x_{i}\right)$ develop discontinuities. These jumps, however, become smaller and eventually disappear as one approaches convergence, resulting in smooth matrix functions $R\left(x_{i}\right), Q\left(x_{i}\right)$ within the unit-cell.

However, the orthonormalization procedure described above introduces a non-trivial gauge change as one moves from one side of the unit-cell to the other. As a result, the matrices $Q\left(x_{i}\right), R\left(x_{i}\right)$ do not trivially connect back to themselves at the end of the unit-cell, even if they originally did. For the purpose of interpolating the matrices a smooth gauge is favourable. To this end, we first canonize the unit-cell MPS by calculating the left and right eigen-vectors $l$ and $r$ of the unit-cell transfer operator

$$
T_{U C}=\sum_{\left\{n_{i}\right\}}\left(A_{n_{1}}^{[1]} \ldots A_{n_{N}}^{[N]}\right) \otimes\left(\bar{A}_{n_{1}}^{[1]} \ldots \bar{A}_{n_{N}}^{[N]}\right)
$$

from which we obtain $X \equiv \sqrt{r}$ and $Y \equiv \sqrt{l}$. We then compute $U \lambda V=Y X$, and absorb $V^{\dagger} X^{-1}$ into the leftmost and $Y^{-1} U \lambda$ into the rightmost MPS matrix. Sweeping from right to left, we then successively orthonormalize the matrices using an SVD on the matrices $A_{n_{i}}^{[i]}=U^{[i]} \lambda^{[i]} V_{n_{i}}^{[i]}$ (indices $[i]$ in square brackets are position labels). To fix the gauge freedom of the SVD, we fix the diagonal of the right isometry $V_{n_{i}}^{[i]}$ to be real and positive. In a successive sweep from the left to the right boundary we fix the diagonal of the matrix $U_{n_{i}}^{[i]}$ in $A_{n_{i}}^{[i]}=U_{n_{i}}^{[i]} \lambda^{[i]} V^{[i]}$ to be real and positive. The right boundary matrix $V^{[N]}$ will then be a diagonal unitary matrix which can be distributed over the unit-cell:

$$
e^{i \mathcal{H} L} \equiv V^{[N]}=\prod_{i}^{N} e^{i \epsilon \mathcal{H}} \equiv \prod_{i}^{N} G
$$

where $L$ is the length of the unit-cell, $N$ is the number of lattice points, $\epsilon=L / N$, and $\mathcal{H}$ is a hermitian operator. $G$ is close to the identity and can be written as $G=\mathbb{1}+\epsilon \tilde{\mathcal{H}}$, with $\tilde{\mathcal{H}}$ an almost hermitian operator. $V^{[N]}$ can now be equally distributed over all matrices by the following two steps: First, at every site $i \in\{1 \ldots N\}$ transform the matrices $Q\left(x_{i}\right), R\left(x_{i}\right)$ according to

$$
\begin{aligned}
& Q\left(x_{i}\right) \leftarrow\left(G^{\dagger}\right)^{i-1} Q\left(x_{i}\right) G^{i-1} \\
& R\left(x_{i}\right) \leftarrow\left(G^{\dagger}\right)^{i-1} R\left(x_{i}\right) G^{i-1} .
\end{aligned}
$$



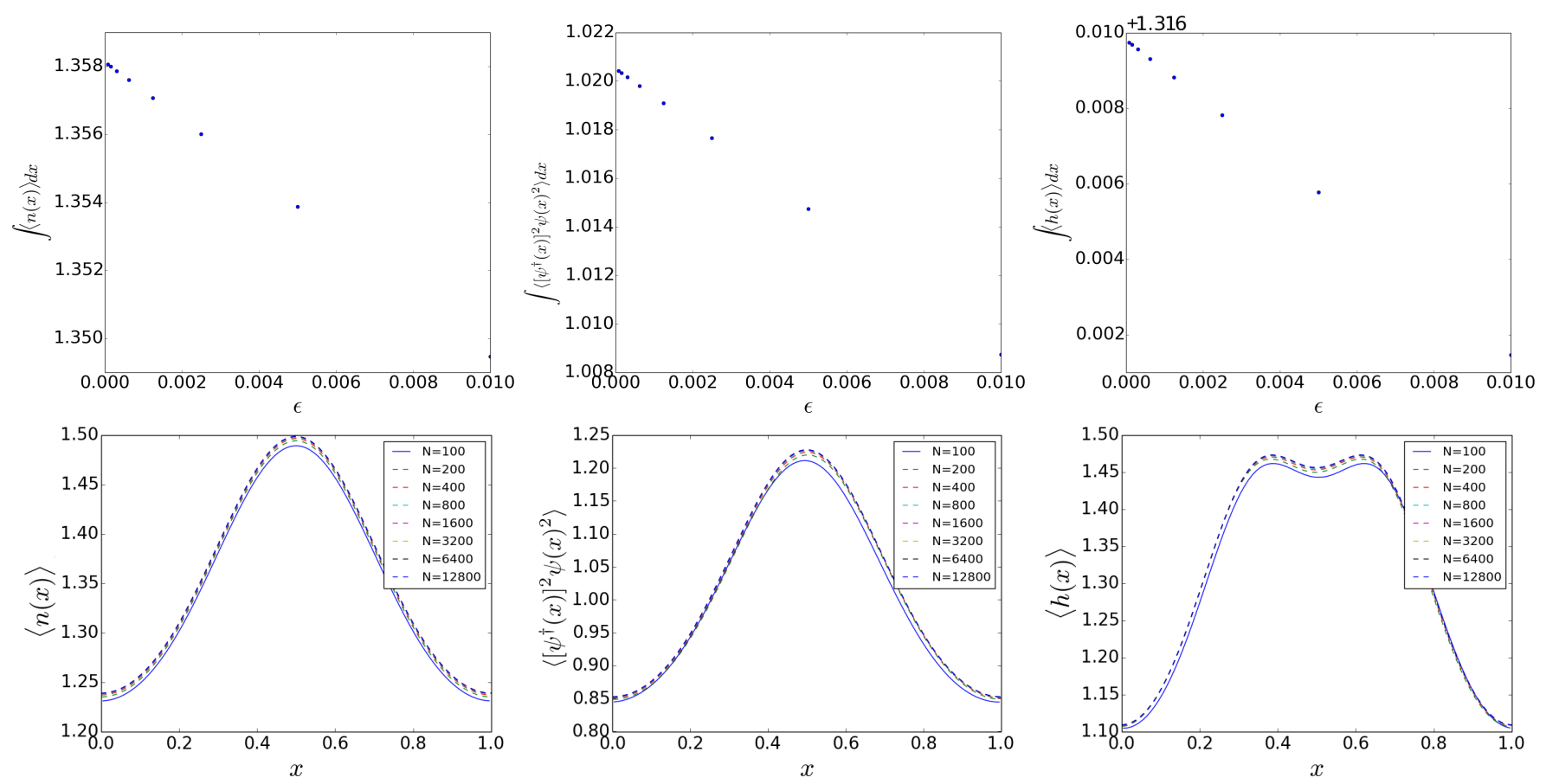

FIG. A1: Convergence of interpolation scheme for different final grid-spacings. We start with a ground-state on a grid of $N=100$ sites per unit-cell and interpolate it, using spline interpolation, to different final grids (see legend). The upper panel (from left to right) shows the total particle number $\int_{0}^{L}\langle n(x)\rangle d x$, the total interaction energy $\int_{0}^{L}\left\langle\left[\psi^{\dagger}(x)\right]^{2} \psi^{2}(x)\right\rangle d x$ and total energy $\int_{0}^{L}\langle h(x)\rangle d x$ (see main text) as a function of grid spacing $\epsilon$. Each shows linear dependence on $\epsilon$. Lower panel: spatially resolved $\langle n(x)\rangle,\left\langle\left[\psi^{\dagger}(x)\right]^{2} \psi^{2}(x)\right\rangle$ and $\langle h(x)\rangle$ for different number $N$ of final lattice points per unit-cell.

Second, transform each matrix

$$
\begin{array}{r}
Q\left(x_{i}\right) \leftarrow Q\left(x_{i}\right)+\tilde{\mathcal{H}}+\epsilon Q\left(x_{i}\right) \tilde{\mathcal{H}} \\
R\left(x_{i}\right) \leftarrow R\left(x_{i}\right)+\epsilon R\left(x_{i}\right) \tilde{\mathcal{H}}
\end{array}
$$

The resulting matrices $Q\left(x_{i}\right), R\left(x_{i}\right)$ will connect smoothly back to themselves.

\section{Appendix B: Continuous limit of fine-graining}

In this section we explore in more detail how interpolation affects different observables. In particular, we will focus on the observables $\langle n(x)\rangle,\left\langle\left[\psi^{\dagger}(x)\right]^{2} \psi^{2}(x)\right\rangle$ and $\langle h(x)\rangle$ (see also main text). We first obtain the ground-state of Eq. (46) for $D=16, \mu_{0}=-0.5, V_{0}=-1.0, g=1.0$ and $N=100$ sites per unit-cell. We then interpolate the corresponding matrices $Q\left(x_{i}\right), R\left(x_{i}\right)$ to different finer grids with $N=200,400,800,1600,3200,6400$ and 12800 sites per unit-cell, and calculate the observables $\langle n(x)\rangle,\left\langle\left[\psi^{\dagger}(x)\right]^{2} \psi^{2}(x)\right\rangle$ and $\langle h(x)\rangle$ after normalizing the state. The results are shown in Fig. A1. The upper panel shows $\int_{0}^{L} d x\langle n(x)\rangle, \int_{0}^{L} d x\left\langle\left[\psi^{\dagger}(x)\right]^{2} \psi^{2}(x)\right\rangle$ and $\int_{0}^{L} d x\langle h(x)\rangle$, integrated over the unit-cell, as a function of $\epsilon$. We observe a clear linear dependence on $\epsilon$ as we decrease the lattice spacing. The lower panel shows the spatially resolved quantities for different $N$.

\section{Appendix C: Short range entanglement}

In Fig. 4 in the main text we show how the entanglement entropy $S(l)$ of a region of size $l$ in the center of the unit-cell ( for parameters $D=16, \mu_{0}=-0.5, V_{0}=$ $-1.0, g=1.0$, see Fig. 33. As pointed out in the main text we observe a transition from a power law increase for small $l$ to a less pronounced increase for larger $l$ at a certain length scale $\xi \approx 0.05$. Fig. A2 shows $S(l)$ for two different locations of the region $l$, namely in the center of the unit-cell and close to the left boundary of the unitcell (with $l$ growing to the right). The plot illustrates that $\xi$ varies along the unit-cell, which could be used as basis for determining grid spacings for non-homogeneous discretizations as e.g. shown in Fig. 1 in the main text.

\section{Appendix D: Optimization of periodic MPS on a lattice}

In this section we give a detailed description of the optimization method used to obtain the ground-state of a Hamiltonian $H\left(\epsilon_{\alpha}\right)$ (see main text). We employ the infinite DMRG (iDMRG) method as introduced by McCulloch 44 , extended to the case of a large unit-cell with $N \gg 2$ sites. To make the document self-contained, we give in the following a detailed explanation of the al- 


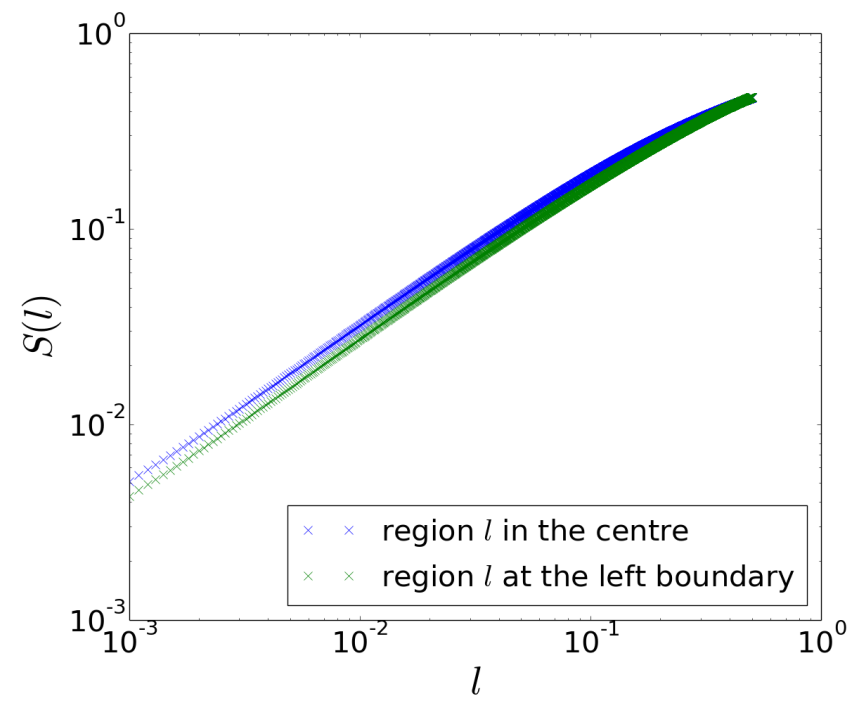

FIG. A2: Entanglement entropy $S(l)$ as a function of length $l$. Entanglement entropy $S(l)$ of region of length $l$ in the ground-state of Eq. (46) for $D=16, \mu_{0}=-0.5, V_{0}=$ $-1.0, g=1.0, L=1.0$, and $N=10^{4}$ lattice points per unitcell. We show $S(l)$ for two different regions $l$ : blue crosses show $S(l)$ as a function of $l$ for $l$ starting at the center of the unit-cell, and green crosses show $S(l)$ for $l$ starting close to the left boundary. For the latter case we see that $S(l)$ departs from the linear behaviour at a larger length scale than for $l$ in the center of the unit-cell. This fact could be used devise non-homogeneous discretization schemes for MPS that use a varying grid spacing based on this length scale. Note that the slope of both curves is very similar, which could suggest a universal short lengths scale behaviour of the entanglement entropy.

gorithm. For the sake of simplicity, we restrict ourselves to the case of $N=4$. Results for arbitrary $N$ follow straightforwardly.

Before going into more detail, we first introduce some necessary diagrammatic notations for MPS. In the following we consider periodic MPS with $N$ tensors $\left\{A^{[1]}, \ldots, A^{[N]}\right\}$ per unit-cell. Such a state is pictorially represented as

$$
|\Psi\rangle=\cdots-\underbrace{1_{j}}_{1}-2_{i}-3_{j}-4_{j}-\cdots
$$

where dots indicate an infinite repetition of the unit-cell. Subscripts $j \in \mathbb{Z}$ label the unit-cell. For brevity, we will in the following omit dots and subscripts $j$ if no confusion can arise. Using standard regauging techniques ${ }^{45}$ (see Appendix A, the state can be regauged into left and right orthogonal forms

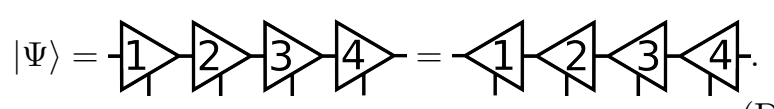

The left and right orthogonal tensors $-i>$ and $<$ i obey

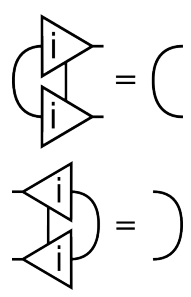

(see also Eq.(30)).

Of particular use is the so-called canonical form ${ }^{45}$ of an MPS, given by the following decomposition:

$$
|\Psi\rangle=\langle 1\rangle-\hat{Y}\rangle-\hat{3}\rangle-(3)-(4) \text {. }
$$

-(i) are diagonal bond matrices containing the Schmidt values $\lambda_{\alpha}$ on bond $i$, and $\Gamma_{\alpha \beta}^{n,[i]} \equiv\langle$ is a $D \times D \times d$ tensor at site $i$. In our convention, the index label $i$ of a bond matrix can be deduced from the index of the left tensor next to it. For the sake of brevity, we will thus omit it if no confusion can arise. We will use angle brackets to denote inverses of matrices, i.e. $[0]$ is the inverse of $-\mathrm{O}$, with $\mathrm{O}+\mathrm{O}-\mathrm{O}-\mathrm{O}=-\equiv \mathbb{1}$. The canonical form is particularly useful because the left and right orthogonal form of the MPS can be easily extracted using the relations

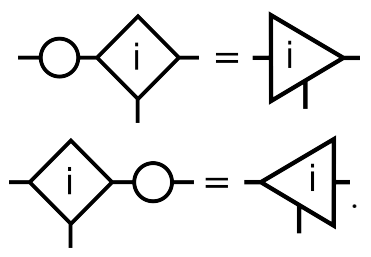

Finally, we introduce the parallel decomposition $\underline{46}$ $|\Psi\rangle=$

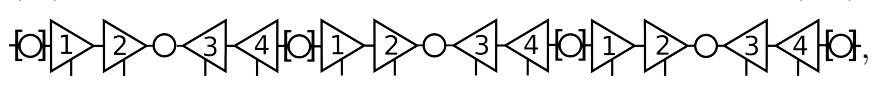

obtained e.g. from the canonical form by inserting identities 1070 - and using Eqs. D6 and (D7). Each unit-cell is here decomposed into a set of left and right orthogonal tensors, with matrices $-\mathrm{O}$ and $[\mathrm{O}]$ between the connection points. The positions of the matrices - - in Eq. (D8) are called the orthogonality centers. The matrices $-\mathrm{O}$ - can be shifted inside the unit-cell using a QR or SV decomposition,

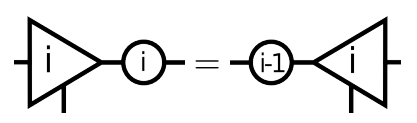

In particular, they can be shifted to the unit-cell boundaries, where they can be absorbed into [O]. A defining 
property of the parallel decomposition is that this absorption results in 14 - (4) $=U_{l}$ and -10 - $U_{r}$, with unitary matrices $U_{l}, U_{r}$. This implies that within each unit-cell local truncation of the MPS bond-dimension can be done optimally. The parallel decomposition is also central to the parallel version of DMRG on finite lattices 46 .

In the following we explain the different steps involved in the ground-state optimization of a periodic Hamiltonian $H$ in the thermodynamic limit. At each step of the optimization, the state is represented by $N$ unitcell tensors $\left\{A^{[1]}, \ldots, A^{[N]}\right\}$ which can be patched into an infinite MPS. Fig. A3 gives an overview of the first five steps of the algorithm. Different colours of matrices and tensors indicate an update. In Fig. A3 (a), the MPS is initialized with $N$ random tensors per unit-cell and brought into the parallel decomposition (red tensors and matrices in Fig. A3 (a)). The initial matrices are diagonal, containing the Schmidt values. The tensors of each unit-cell are then updated using standard variational optimization techniques for MPS (see below for details), see Fig. A3 (b). The updated objects are shown in green, and updates are identical between each unit-cell. Note that the matrices - - are dense. Between each unit-cell resides a matrix 1 from the previous step. (c) A resolution of identity is inserted. Using the $\mathrm{QR}$ or SV decomposition, the two matrices at the orthogonality centers are shifted left, respectively right (see Eq. D9), until the boundary matrix reached (Fig. A3 (d)). The three matrices -4i- (4iare contracted into a new matrix - - (see Fig. A3 (a')). The state is now in what we call quasi-parallel decomposition with respect to a new unit-cell that has been shifted by $N / 2$. Quasi-parallel refers to the fact that $[0]$ (2i)and 27 - 7 are in general both non-unitary. Note that during the optimization, the matrices will converge to unitary matrices. The degree of unitarity of the matrices [O] (2i) and - 27- can be used as an alternative measure of convergence. This completes the first iteration of the algorithm. In the next iteration, the tensors of the new unit-cell $\{N / 2+1, \ldots N, 1 \ldots N / 2\}$ are then going to be updated. Over time, several slightly different variants of how to proceed at this point have been developed. We will below explain three different approaches, which we find the most convenient one for our case. Before, however, let us first explain in more detail the individual steps in Fig. A3.

We start with the update from (a) to (b). Within each unit-cell, we use standard variational MPS optimization $^{214}$ for a finite system to update the tensors. For a given, fixed unit-cell, the updated tensors $\left\{\tilde{A}^{[1]}, \ldots, \tilde{A}^{[N]}\right\}$ are found from minimizing the expectation value of the energy with respect to the unit-cell

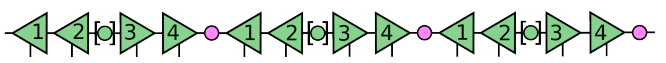

FIG. A3: Outline of the first steps of an iDMRG calculation. (a) Initial state in parallel decomposition. (b) State after all unit-cell tensors have been updated. (c) An identity resolution is inserted at the center of each unit-cell. (d) The tensors -1 are moved to the left respectively right unit-cell boundaries. (a') The two matrices - $10-$ are contracted into $-\mathrm{O}$.

tensors, i.e.

$$
\left\{\tilde{A}^{[1]}, \ldots, \tilde{A}^{[N]}\right\}=\underset{\left\{A^{[1]}, \ldots, A^{[N]}\right\}}{\operatorname{argmin}} \frac{\langle\Psi|H| \Psi\rangle}{\langle\Psi \mid \Psi\rangle},
$$

while keeping all tensors outside the unit-cell fixed. Sweeping from left to right, i.e. $i=1 \ldots N$, the minimization proceeds by optimizing one tensor $A^{[i]}$ at a time. For each site $i$, this minimization leads to a sparse eigenvalue equation with an effective Hamiltonian $H_{\text {eff }}$. For example, for the initial optimization of site $i=1, H_{\text {eff }}$ has the form

$$
H_{e f f}=\cdots+
$$

The unit-cell tensors are highlighted in red, and we have used a Matrix Product Operator representation ${ }^{2}$ (see Appendix E below) of the Hamiltonian Eq. 46,

$$
H=\cdots-\frac{1}{1}+\frac{1}{3}-\frac{1}{4}-\cdots
$$

$C_{\alpha \beta}^{n n^{\prime}} \equiv-\overbrace{1}^{1}-$ is a sparse four-leg tensor (see Appendix $\mathrm{E}$ below). Using standard contraction techniques for MPS (Appendix E) the infinite tensor network in Eq. D11) can be contracted, resulting in the expression

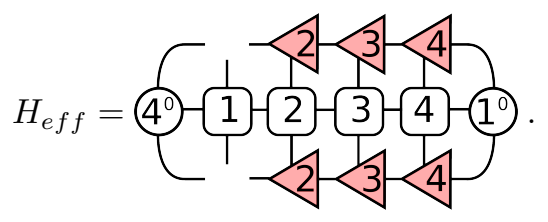

We call (40- and -(1) the effective left and right unit-cell environments. The labels indicate the index of the last 
MPS tensor that has been contracted into $(40-$ and $-(10)$. The superscripts are the iDMRG iteration number. (40is thus the left environment of tensor $i=1$ at iteration $q=0$. (and likewise for -(1)). An updated $\tilde{A}^{[i]} \equiv-i$ is obtained from the (properly reshaped and normalized) lowest eigen-vector of $H_{\text {eff }}$. Using a QR or SV decomposition, it is decomposed into

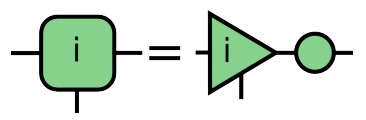

and inserted back into the MPS. One then moves one site to the right, where the above procedure is repeated with

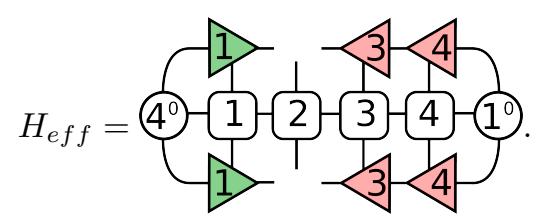

The tensors left and right environments of site $i=2$. The optimization keeps sweeping right and left (using a similar update method for the left sweep) until sufficient convergence is reached (we only sweep once from left to right for our applications). The orthogonality center is then moved to the central bond, where a resolution of the identity - 107 is inserted. The matrices - are shifted to the left and right boundary of the unit-cell (see Fig. A3 (c) and (d)) where they are contracted into $-\mathrm{O}=-4 \mathrm{O}-\mathrm{O}$ - (see Fig. $\mathrm{A3}$ $\left.\left(a^{\prime}\right)\right)$. The state is now in a quasi-parallel decomposition with respect to a unit-cell that has been shifted by $N / 2$. Note, however, that it is no longer in its canonical form. The next iteration consists of updating the tensors $\left\{\tilde{A}^{[N / 2+1]}, \ldots, \tilde{A}^{[N]}, \tilde{A}^{[1]}, \ldots \tilde{A}^{[N / 2]}\right\}$ of this shifted unit-cell. To this end, one needs to calculate an effective Hamiltonian for the shifted unit-cell. Several slightly different methods can be used to proceed at this point. In the following, we will explain two of them in more detail.

In the iDMRG algorithm, the calculation of a new effective Hamiltonian is achieved by recycling the left and right unit-cell environments calculated during the previous iteration. The left and right unit-cell environments at iteration $q=1,\left(2 \mathrm{2}\right.$ - and $-3^{3}$, in this case are given by

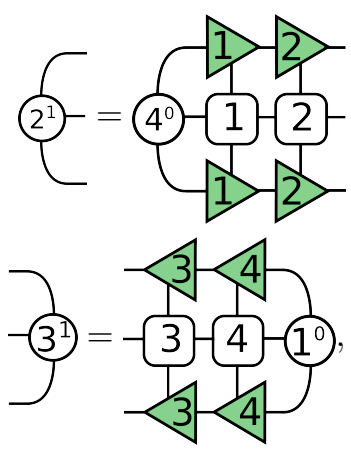

The effective Hamiltonian for e.g. site $i=3$ during the second iteration $q=1$ assumes the form

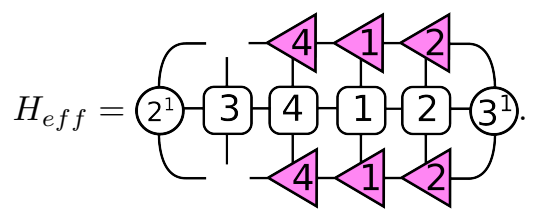

We have here highlighted the currently optimized unitcell tensors in pink. From here on the method proceeds by repeatedly cycling through the steps (b)-(a') of Fig. A3, and updating the left and right unit-cell environments as described above.

While this method works well in general, an inconsistency arises after the first iteration: the unit-cell environments in Eqs. (D16) and (D17) are calculated by adding updated tensors to (40- and -19. These two, however, have both been obtained from contracting an infinite tensor network containing the tensors of the initial MPS. They do not contain any information of the update and are thus different from the ones that would be obtained using the current MPS (which has been updated within each unitcell). Thus, instead of recycling the environments after each iteration, one could also regauge the MPS into its canonical form and recalculate the effective left and right unit-cell environments. This approach is seen to result in slightly lower energies of the optimized MPS.

For the case of $N=1$, a very similar approach has been proposed for the setting of homogeneous cMPS 32 , termed gradient optimization for cMPS. The gradient optimization for homogeneous lattices with $N=1$ can be obtained by slightly tweaking the above method, as we will now describe in the following. Consider a state with $N=1$ in its parallel decomposition, i.e.

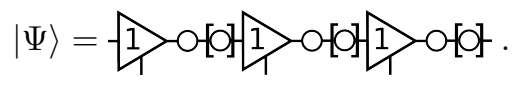

The effective unit-cell Hamiltonian for this state is given 
by

$$
H_{e f f}=\cdots \text { (1) }
$$

The update of the tensor 1 - -1 - proceeds now in a different manner than described above. Instead of finding an approximate lowest eigen-vector of Eq. D20 we calculate a local gradient

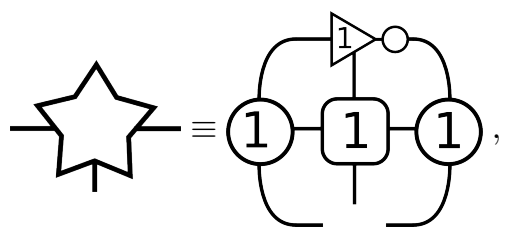

and use it to update the tensor:

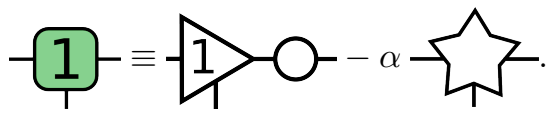

$\alpha$ is a small positive number. If $\alpha$ is chosen such as to minimize the energy expectation value

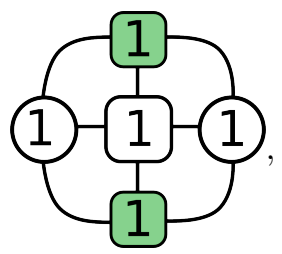

the update is equivalent to finding the lowest energy state in the Krylov space spanned by $\{|\hat{1}\rangle-0,|\widehat{r}\rangle\}$. After this update, the state is in the form

$$
|\Psi\rangle=-1 \text { 다 } 1 \text { 다 } 1 \text { 다. }
$$

The matrices 107 are now absorbed back into 1 from the right hand side, and the iteration is restarted. A related approach for $1 \mathrm{~d}$ lattice models which avoids the possibly ill-condition inversion 10 has recently been proposed by Zauner-Stauber et al. $\stackrel{47}{ }$. For the case of cMPS, the gradient optimization greatly outperforms other optimization approaches.

As we have found numerically, recalculating the full environments gives more accurate results for ground-state energies than recycling the environments. However, for large $N \gg 1$, it is slower than standard iDMRG due to the necessary recalculation of the environments. For the model considered in this manuscript, the difference in accuracy between the two approaches is small enough that in present manuscript we use the simple recycling method for all calculations. In order to facilitate the extraction the cMPS content of the optimized lattice MPS, we use slightly modified techniques for shifting the orthogonality center and regauging the state, as described in detail in Appendix $\mathrm{A}$

\section{Appendix E: Contracting Eq. D11}

In this Appendix we discuss in detail how to contract the infinite tensor network appearing in Eq.(D11). In the following we discuss the case of a nearest neighbor Hamiltonian. The extension to more general Hamiltonians follows straightforwardly. Consider the case of a Hamiltonian of the form

$$
H=\sum_{i \in \mathbb{Z}} h_{i, i+1}=\sum_{i \in \mathbb{Z}} \stackrel{+i+1,}{i+1}
$$

where we have introduced a diagrammatic notation for the two-site operators $h_{i, i+1}$. We assume further a periodicity of the Hamiltonian over $N=4$ sites (see main text), such that we can decompose Eq.(E1) into

$$
\begin{aligned}
H & =\sum_{j \in \mathbb{Z}} H_{j} \\
H_{j} & =\sum_{i=1}^{4} h_{i, i \oplus 1}=\sum_{i=1}^{4}+i \frac{1}{i},
\end{aligned}
$$

with $j$ running over the unit-cells, and $i \oplus 1 \equiv i \bmod N+1$. Using standard techniques ${ }^{2}, H$ can be written in an MPO decomposition

$$
H=\cdots-1
$$

where numbers label sites $i$ inside the unit-cell. Horizontal lines denote auxiliary indices (with a bond-dimension $M)$, and vertical lines are physical indices of the MPO. For example, for the Hamiltonian Eq.46), a possible, non-unique MPO decomposition is given by

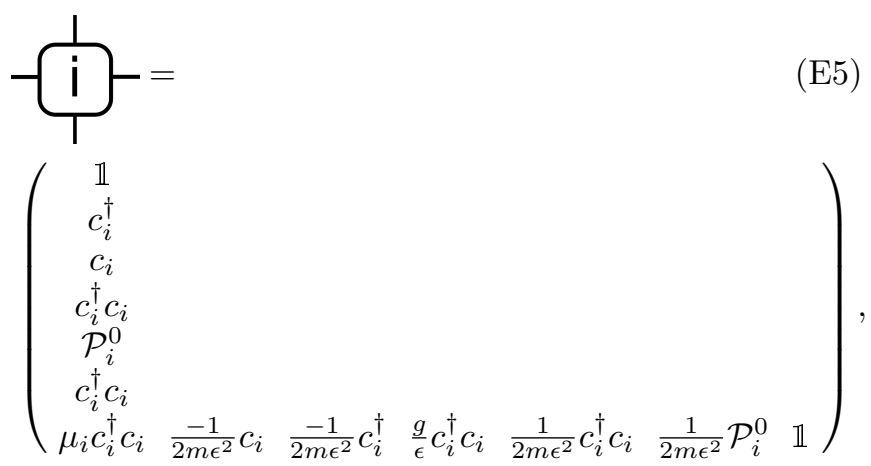

where we have only written entries different from 0 . The MPO bond dimension is in this case $M=7$. For later 
reference we introduce the boundary MPO tensors

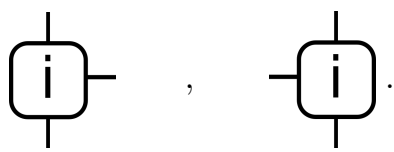

In our MPO convention, 1 is given by the last column of Eq. E5 , and $-\frac{1}{\mathrm{i}}$ is given by its first row. We refer the reader to the literature ${ }^{2}$ for a detailed introduction to the MPO formalism.

Written out explicitly for the case $N=4$ we have

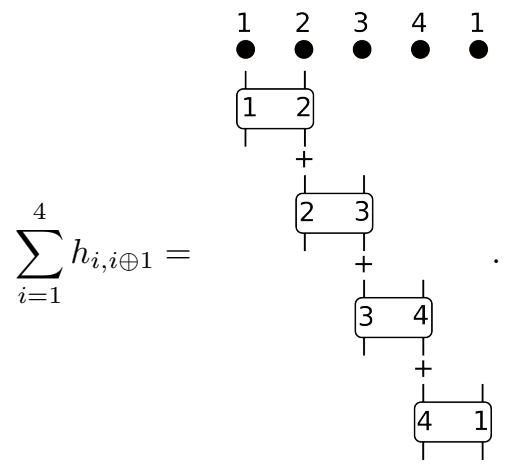

For a fixed unit-cell we will now calculate the right unitcell environment (see main text)

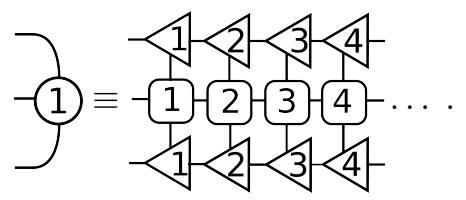

For an MPO with e.g. bond dimension $M$, this would be a vector of $M D \times D$ matrices. In vector notation, it is

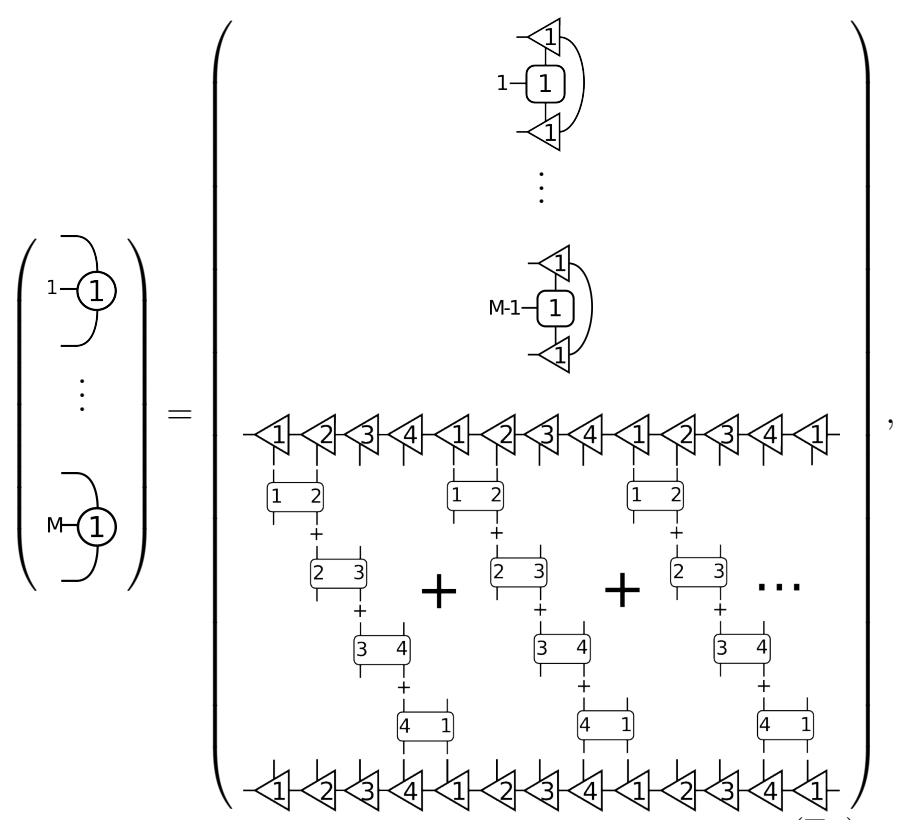

(E9) where we have explicitly written out the vector index. Using an MPO decomposition of the unit-cell Hamiltonian,

$$
\begin{array}{ll}
1 & 2
\end{array}+2 \quad 3+3+4+4=4
$$

we can rewrite the last component of Eq. E24 as

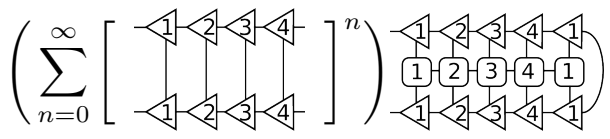

with the right-normalized unit-cell transfer operator

$$
\mathbb{E}_{r} \equiv \underbrace{1}_{12} \sqrt[3]{4}
$$

and the renormalized unit-cell Hamiltonian

$$
\left|h_{r}\right\rangle=\begin{array}{lllll}
1 & 2 & 3 & 4 & 1 \\
1 & -2 & -3 & -4 & -1 \\
1 & 2 & 3 & 4 & 1
\end{array} .
$$

The operator $\mathbb{E}_{r}$ has a left and right dominant eigenvector $\langle l|,| \mathbb{1}\rangle$ to eigenvalue $\eta=1$. Thus, the geometric series in Eq. E11) diverges. This can be cured by restricting the operator $\mathbb{E}_{r}$ to the orthogonal complement of the subspace $|\mathbb{1}\rangle\langle l|$, i.e. by the replacements

$$
\begin{aligned}
\mathbb{E}_{r} & \rightarrow \mathbb{E}_{r \perp}=\mathbb{E}_{r}-|\mathbb{1}\rangle\langle l| \\
\left|h_{r}\right\rangle & \rightarrow\left|h_{r}\right\rangle_{\perp}=\left|h_{r}\right\rangle-\left\langle l \mid h_{r}\right\rangle|\mathbb{1}\rangle
\end{aligned}
$$

The geometric series Eq. E11 transforms into

$$
\left|H_{r}\right\rangle_{\perp} \equiv\left(\sum_{n=0}^{\infty}\left[\mathbb{E}_{r \perp}\right]^{n}\right)\left|h_{r}\right\rangle_{\perp}=\frac{1}{\mathbb{1}-\mathbb{E}_{r \perp}}\left|h_{r}\right\rangle_{\perp}
$$

which can be solved iteratively for $\left|H_{r}\right\rangle_{\perp}$ using sparse solvers like e.g. the lgmres method from the scipy package.

The approach for calculating the left unit-cell environment starts from the expression

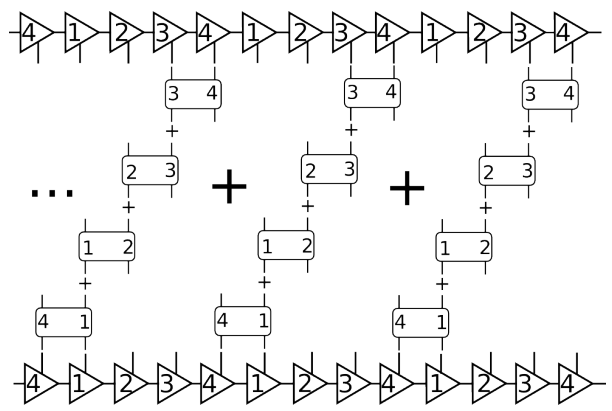

and then follows the same steps, using an MPO decomposition for the unit-cell Hamiltonian of the form

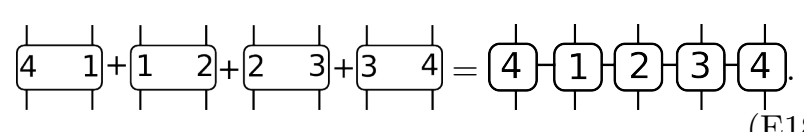


With the definitions

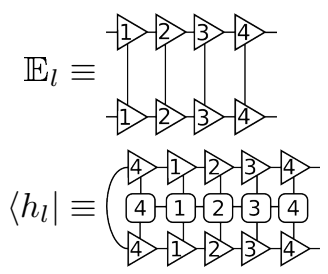

and the replacements

$$
\begin{aligned}
\mathbb{E}_{l} & \rightarrow \mathbb{E}_{l \perp}=\mathbb{E}_{l}-|r\rangle\langle\mathbb{1}| \\
\left\langle h_{l}\right| & \rightarrow\left\langle\left. h_{l}\right|_{\perp}=\left\langle h_{l}\right|-\left\langle h_{l} \mid r\right\rangle\langle\mathbb{1}|\right.
\end{aligned}
$$

one obtains

$$
\left\langleH _ { l } | _ { \perp } \equiv \left\langle\left. h_{l}\right|_{\perp} \frac{1}{\mathbb{1}-\mathbb{E}_{l \perp}} .\right.\right.
$$

Here, $\langle\mathbb{1}|$ and $|r\rangle$ are the dominant left and right eigen- vectors of $\mathbb{E}_{l}$. The final result is

$$
\begin{aligned}
& \text { (1) }
\end{aligned}
$$

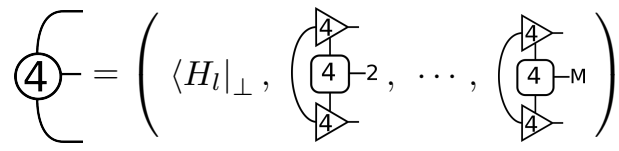

